\title{
Horizontal gene transfer in Histophilus somni and its role in the evolution of pathogenic strain 2336, as determined by comparative genomic analyses
}

Shivakumara Siddaramappa ${ }^{1,2}$, Jean F Challacombe ${ }^{2}$, Alison J Duncan ${ }^{1}$, Allison F Gillaspy ${ }^{3}$, Matthew Carson ${ }^{3}$, Jenny Gipson ${ }^{3}$, Joshua Orvis ${ }^{3}$, Jeremy Zaitshik ${ }^{3}$, Gentry Barnes³, David Bruce², Olga Chertkov², J Chris Detter ${ }^{2}$, Cliff S Han², Roxanne Tapia², Linda S Thompson², David W Dyer ${ }^{3}$ and Thomas J Inzana ${ }^{{ }^{*}}$

\begin{abstract}
Background: Pneumonia and myocarditis are the most commonly reported diseases due to Histophilus somni, an opportunistic pathogen of the reproductive and respiratory tracts of cattle. Thus far only a few genes involved in metabolic and virulence functions have been identified and characterized in $\mathrm{H}$. somni using traditional methods. Analyses of the genome sequences of several Pasteurellaceae species have provided insights into their biology and evolution. In view of the economic and ecological importance of $\mathrm{H}$. somni, the genome sequence of pneumonia strain 2336 has been determined and compared to that of commensal strain 129Pt and other members of the Pasteurellaceae.
\end{abstract}

Results: The chromosome of strain 2336 (2,263,857 bp) contained 1,980 protein coding genes, whereas the chromosome of strain $129 \mathrm{Pt}(2,007,700 \mathrm{bp})$ contained only 1,792 protein coding genes. Although the chromosomes of the two strains differ in size, their average GC content, gene density (total number of genes predicted on the chromosome), and percentage of sequence (number of genes) that encodes proteins were similar. The chromosomes of these strains also contained a number of discrete prophage regions and genomic islands. One of the genomic islands in strain 2336 contained genes putatively involved in copper, zinc, and tetracycline resistance. Using the genome sequence data and comparative analyses with other members of the Pasteurellaceae, several H. somni genes that may encode proteins involved in virulence (e.g., filamentous haemaggutinins, adhesins, and polysaccharide biosynthesis/modification enzymes) were identified. The two strains contained a total of $17 \mathrm{ORFs}$ that encode putative glycosyltransferases and some of these ORFs had characteristic simple sequence repeats within them. Most of the genes/loci common to both the strains were located in different regions of the two chromosomes and occurred in opposite orientations, indicating genome rearrangement since their divergence from a common ancestor.

Conclusions: Since the genome of strain 129Pt was $\sim 256,000$ bp smaller than that of strain 2336, these genomes provide yet another paradigm for studying evolutionary gene loss and/or gain in regard to virulence repertoire and pathogenic ability. Analyses of the complete genome sequences revealed that bacteriophage- and transposonmediated horizontal gene transfer had occurred at several loci in the chromosomes of strains 2336 and 129Pt. It appears that these mobile genetic elements have played a major role in creating genomic diversity and phenotypic variability among the two H. somni strains.

\footnotetext{
* Correspondence: tinzana@vt.edu

${ }^{1}$ Center for Molecular Medicine and Infectious Diseases, Virginia-Maryland Regional College of Veterinary Medicine, Virginia Polytechnic Institute and State University, Blacksburg, Virginia 24061, USA

Full list of author information is available at the end of the article
} 


\section{Background}

Histophilus somni is a commensal or opportunistic pathogen of the reproductive and respiratory tracts of cattle. $H$. somni was initially identified as the etiologic agent of bovine thrombotic meningoencephalitis (TME), but also causes bovine shipping fever pneumonia, either independently or in association with Mannheimia haemolytica and Pasteurella multocida. Pneumonia and myocarditis are currently the most commonly reported diseases due to H. somni [1]. Infections resulting in abortion, infertility, arthritis, septicemia, and mastitis can also be caused by $H$. somni with varying degrees of frequency and severity in cattle [2]. Similar disease conditions associated with strains of $H$. somni have been described in sheep [2]. Relatively less pathogenic and/or avirulent variants of $H$. somni have also been isolated from cattle, most frequently from the mucosal surfaces of the genital tract [3].

Numerous in vitro and in vivo studies during the pregenomic era have shed light on the differences in virulence properties between $H$. somni pathogenic isolates from sick animals and serum-sensitive commensal isolates from the genital tract [4]. However, thus far only a few genes involved in lipooligosaccharide (LOS) biosynthesis and serum-resistance have been identified in $H$. somni using DNA/DNA and DNA/protein comparisons [5-7]. H. somni pneumonia strain 2336 and preputial strain 129Pt have been comprehensively characterized phenotypically and have been analyzed in several comparative studies [8-10]. However, a comprehensive understanding of the genetic basis that determines the phenotypic variability among $H$. somni stains is necessary to gain further insights into their pathogenicity.

Comparative (in silico) analysis of bacterial genomes is a powerful tool for the prediction and/or identification of biochemical differences, virulence attributes, pathogenic ability, and adaptive evolution among related species/ strains [11]. Among the Pasteurellaceae, the genomes of one or more species pathogenic to humans or animals from the genera Actinobacillus, Haemophilus, Mannheimia, Pasteurella, and others have been sequenced. The availability of these genome sequences has facilitated whole genome comparisons that have provided insights into the physiology and pathogenic evolution of the corresponding bacteria [12,13].

Horizontal gene transfer (HGT: defined as the "acquisition of new genes either directly by transformation with naked DNA, transduction with phages, or the uptake of plasmids or chromosomal fragments by conjugation") plays a critical role in driving the evolution of pathogenic bacteria [14]. Reduction in genome size (referred to as reductive evolution) can occur as a result of continuous loss of genetic material due to gene deletion and/or mutation followed by DNA erosion [15]. Previous analyses by biochemical and pulsed field gel electrophoresis indicated that H. somni strains 2336 and 129Pt have common ancestry, but are non-clonal $[16,17]$. The following mechanisms may have engendered the genetic differences between these strains: (i) only one strain acquired genes by HGT while the other one did not; (ii) only one strain lost genes by deletion/mutation and underwent 'reductive evolution'; (iii) both strains independently and continuously acquired and lost genes, and the net loss or gain of genes is a determinant of their divergent evolution; (iv) gene convergence and the accumulation of synonymous and/or nonsynonymous nucleotide substitutions occurred across the genomes of the two strains.

The rationale for the present study was to determine, using whole genome sequencing and comparative genomics, the mechanisms responsible for genetic variability between the two strains. It was also envisaged that a comparative genomics and bioinformatics approach would facilitate identification of $H$. somni genes putatively involved in virulence and pathogenesis.

\section{Methods}

Genomic DNA (2 mg) from H. somni strain 2336 was purified using the Puregene protocol (Gentra Systems, Minneapolis, MN). The shotgun sequencing phase for this genome required $\sim 35,200$ sequence reads to reach 8 -fold coverage [18]. Library construction, template preparation, sequencing, assembly, and data analyses were performed as described previously $[19,20]$. The sequence data assembled with Phred-Phrap were viewed using Consed to assess data quality and design closure experiments. Consed was also used to identify putative repeat regions so that the problems associated with assembling these regions could be resolved by way of combinatorial PCR experiments to isolate the repeat sequences on PCR amplicons. The location and exact sequence of each repeat was confirmed by isolating PCR fragments that contained each repeat in its entirety, followed by primer walking across the PCR product.

For initial gap closure, Single Primer Amplification of Contig Ends (SPACE), which is similar to the single-primer PCR procedure for rapid identification of transposon insertion sites, was used [21]. Additional primers were designed, as necessary, to verify the correct assembly of contigs by confirmatory PCR. Simultaneously, a fosmid library was constructed for scaffolding purposes using the vector pCC1fos (Epicentre Biotechnologies, Madison, WI) with $40 \mathrm{~kb}$ inserts. Sequencing of the fosmids was necessary to close gaps across sequences that occur more than once in the genome, such as those of insertion sequences and ribosomal genes. Gaps that were not closed by SPACE-walking were closed using the sequence of H. somni strain $129 \mathrm{Pt}$ as a scaffold and the reads were 
assembled with parallel phrap (High Performance Software, LLC). Gap closure at this stage was also facilitated by AUTOFINISH [22]. Possible mis-assemblies were corrected with Dupfinisher [23] or transposon bombing of bridging clones [24] using an EZ::TNTM kit (Epicentre). The National Human Genome Research Institute standards for the Human Genome Project (1 error per 10, 000 assembled bases) were followed for $H$. somni to obtain sufficient quality genomic data.

Final automated annotation of the genome of strain 2336 was performed at the Oak Ridge National Laboratory using methods similar to those used to annotate the strain $129 \mathrm{Pt}$ genome [13]. Briefly, protein domains were identified by comparing each predicted protein against a Hidden Markov Model protein family database [25]. To estimate the number of proteins specific to each strain, the SmithWaterman algorithm [26] was used to compare all predicted proteins from strain 129Pt against those from strain 2336 and vice-versa. Proteins deemed to be specific to each strain were compared against the NCBI non-redundant protein database to determine whether they were hypothetical or conserved hypothetical. The translated ORF was named a hypothetical protein if there was less than $25 \%$ identity or an aligned region was less than $25 \%$ of the predicted protein length. Prediction of the number of subsystems and pairwise BLAST comparisons of protein sets within strains 2336 and $129 \mathrm{Pt}$ were carried out with the Rapid Annotation using Subsystems Technology (RAST), which is a fully automated, prokaryotic genome annotation service [27]. This platform identifies tRNA and rRNA genes using the tools tRNAscan-SE and "search for_rnas", respectively [27].

Multiple genome comparisons were performed using the 'progressive alignment' option available in the program MAUVE version 2.3.0 [28,29]. Default scoring and parameters were used for generating the alignment. A synteny plot was generated using the program NUCmer, which creates a dot plot based on the number of identical alignments between two genomes [30]. Prophage regions (PRs) were identified using Prophinder http:// aclame.ulb.ac.be/Tools/Prophinder/, an algorithm that combines similarity searches, statistical detection of phage-gene enriched regions, and genomic context for prophage prediction [31]. Identification and annotation of genomic islets/islands (GIs) other than prophages were performed based on sequence composition bias and comparative genomic analysis [32]. Briefly, differences in the GC content, the occurrence of 'cornerstone genes' (e.g., transposases), and/or a continuous stretch of genes encoding hypothetical proteins were used as reference points for detection of GIs. Insertion sequences (ISs) were identified by whole genome BLASTX analysis of strains 2336 and 129Pt using the IS finder http:// www-is.biotoul.fr/. Gene acquisition and loss among the two strains was determined by comparing gene order, orientation of genes (forward/reverse), GC content of genes (percent above or below whole genome average), features of intergenic regions (e.g., remnants of IS elements, integration sites), and the similarity of proteins encoded by genes at a locus of interest (> 90\% identity at the predicted protein level). Putative horizontally transferred genes (HTGs; defined as genes whose greatest homology, based on BLASTP scores, is to genes from a more distant phylogenetic group than to genes from the same or a close phylogenetic group as the query genome) were compiled using the integrated microbial genomes (IMG) system http://img.jgi.doe.gov. This system uses not only the best hit (i.e., the homolog with the highest bitscore), but also all the matches that have bitscores equal to or greater than that of the best hit to identify putative HTGs [33]. DNA and protein sequences were aligned using the ClustalW http://www. ebi.ac.uk/Tools/clustalw2/index.html and BOXSHADE http://www.ch.embnet.org/software/BOX_form.html programs as described previously [34].

\section{Results}

\section{Properties of the chromosomes}

The size of the $H$. somni strain 2336 chromosome was $2,263,857 \mathrm{bp}$, and was larger than the chromosomes of the related bacteria Haemophilus ducreyi strain $35000 \mathrm{HP}$ (1,698,955 bp), Haemophilus influenzae strain Rd KW20 (1,830,138 bp), H. influenzae strain 86-028NP $(1,913,428$ bp), H. somni strain $129 \mathrm{Pt}(2,007,700 \mathrm{bp})$, Neisseria gonorrhoeae strain FA 1090 (2,153,922 bp), Neisseria meningitidis serogroup A strain Z2491 (2,184,406 bp), and P. multocida strain Pm70 (2,257,487 bp). However, the chromosome of $H$. somni strain 2336 was smaller than that of Haemophilus parasuis SH0165 (2,269,156 bp), N. meningitidis serogroup B strain MC58 (2,272,360 bp), Actinobacillus pleuropneumoniae L20 (2,274,482 bp), Aggregatibacter aphrophilus NJ8700 (2,313,035 bp), Mannheimia succiniciproducens strain MBEL55E (2,314,078 $\mathrm{bp})$, and M. haemolytica strain BAA-410 ( 2,569,125 bp, draft sequence). Although the chromosomes of the two H. somni strains differ in size by $\sim 256,000 \mathrm{bp}$, their average GC content, gene density (total number of genes predicted on the chromosome), and percentage of the sequence (number of genes) that encodes proteins were similar (Table 1). H. somni strain 2336 did not contain plasmids, but $H$. somni strain $129 \mathrm{Pt}$ contained a single plasmid [34]. Some of the other relevant features of the chromosomes of $H$. somni strains 2336 and $129 \mathrm{Pt}$ are shown in Table 1.

Whole genome alignment using MAUVE showed the presence of extensive blocks of homologous regions, 
Table 1 Characteristics of the genomes of H.somni strains

\begin{tabular}{lll}
\hline Genome & Strain 2336 & Strain 129Pt \\
\hline Chromosome size & 2263857 bp & 2007700 bp \\
Number of protein coding genes & 1980 & 1792 \\
Overall coding density & $88.56 \%$ & $89.6 \%$ \\
*Number of subsystems & 247 & 240 \\
G+C content & $37.38 \%$ & $37.19 \%$ \\
$5 S$ ribosomal RNA genes & 6 & 6 \\
$16 S$ ribosomal RNA genes & 5 & 5 \\
$23 S$ ribosomal RNA genes & 5 & 5 \\
Number of tRNA genes & 49 & 49 \\
Plasmids & None & pHS129 (5178 bp) [34] \\
Prophage regions & 4 & 1 \\
Genomic islands & 3 & 6 \\
GenBank accession number & {$[G e n B a n k: C P 000947]$} & [GenBank:CP000436] \\
\hline
\end{tabular}

*Subsystems predicted by RAST server [27].

which is typical of closely related genomes (Figure 1). To further dissect their co-linearity, a BLASTN comparison of the two genomes was performed at the Joint Genome Institute web site. This analysis indicated that there were 400 homologous regions (219 plus/plus and 181 plus/ minus, sequence range $>1,000 \mathrm{bp}$, but $<30,000 \mathrm{bp}$ ) and the average nucleic acid identity among these homologous regions was $98.5 \%$ (the identity range was $94 \%-99 \%$, E-value $=0$ ). The plus/plus homologous regions refer to those present on the forward strand in both strains (i.e., those that have the same orientation in both the chromosomes), and the plus/minus homologous regions refer to those present on the reverse strand in strain 129Pt in relation to those present on the forward strand in strain 2336 (i.e., those that have the opposite orientation, indicative of chromosome inversion). Several large gaps, translocations, and inversions became visible in the alignment generated by NUCmer (Figure 2). Detailed sequence examination revealed that some of these gaps and/or inversions were associated with integrative and conjugative elements.

\section{Comparison of prophage regions and genomic islands}

Prophinder predicted four PRs in strain 2336, but only one in strain 129Pt (Table 2). PR I of strain 2336 was the shortest and had homology to 3 segments totaling $~ 4,000$ bp of bacteriophage HP1 of $H$. influenzae (66-68\% nucleotide identity, E-value $=0$ to $4 \mathrm{e}-07)$. The GC content of all four PRs from strain 2336 (PR I, 40.23\%; PR II, 43.95\%; PR III, 39.63\%; PR IV, 39.85\%) was higher than that of the overall genome (37.38\% GC). PR II of strain 2336, which had the highest GC content (43.95\%) among the four PRs, also contained a 5,679 bp segment $(774,075$ bp to 779,754 bp) with homology to a region within the genome of $H$. influenzae strain 10810 (72\% nucleotide identity, E-value = $0)$. PR III of strain 2336 was the longest and contained 38 ORFs of unknown function (annotated as encoding hypothetical proteins). The genome of $H$. parasuis strain SH0165 contained several short sequences that had homology to this region (e.g., 5 segments totaling $\sim 2,700$ bp in the $1,136,975$ bp to $1,143,731$ bp region, $69-76 \%$ nucleotide identity, E-value $=5 \mathrm{e}-152$ to $7 \mathrm{e}-11)$. PR IV of strain 2336 was the most conspicuous and contained at

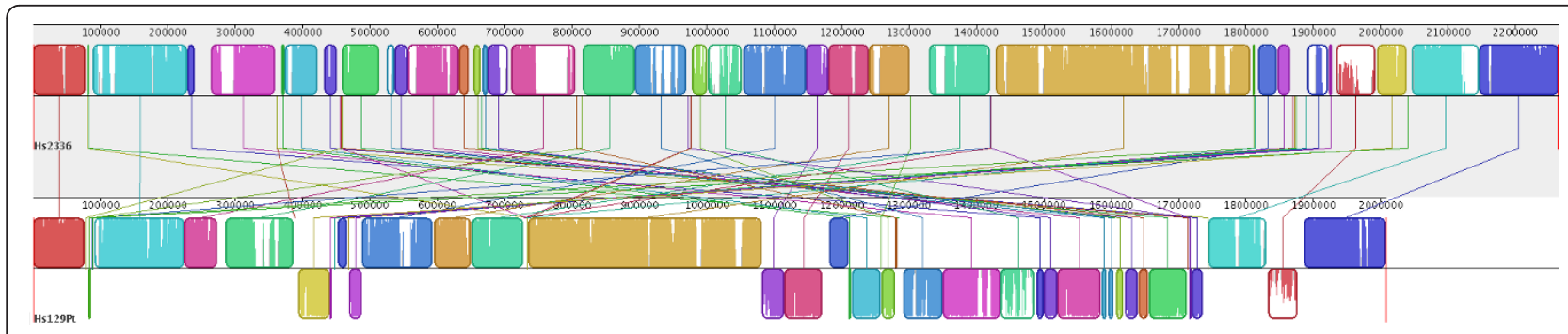

Figure 1 Alignment of the chromosomes of strains 2336 (top) and 129Pt (bottom) using MAUVE 2. Identically colored boxes, known as locally collinear blocks (LCBs), depict homologous regions in the two chromosomes. The edges of LCBs indicate chromosome rearrangements due to recombination, insertions, and/or inversions. Sequences of strain 129Pt inverted in relation to those of strain 2336 are shown as blocks below the horizontal line. The vertical lines connect regions of homology among the two chromosomes. Numbers above the maps indicate nucleotide positions within the respective chromosomes. 


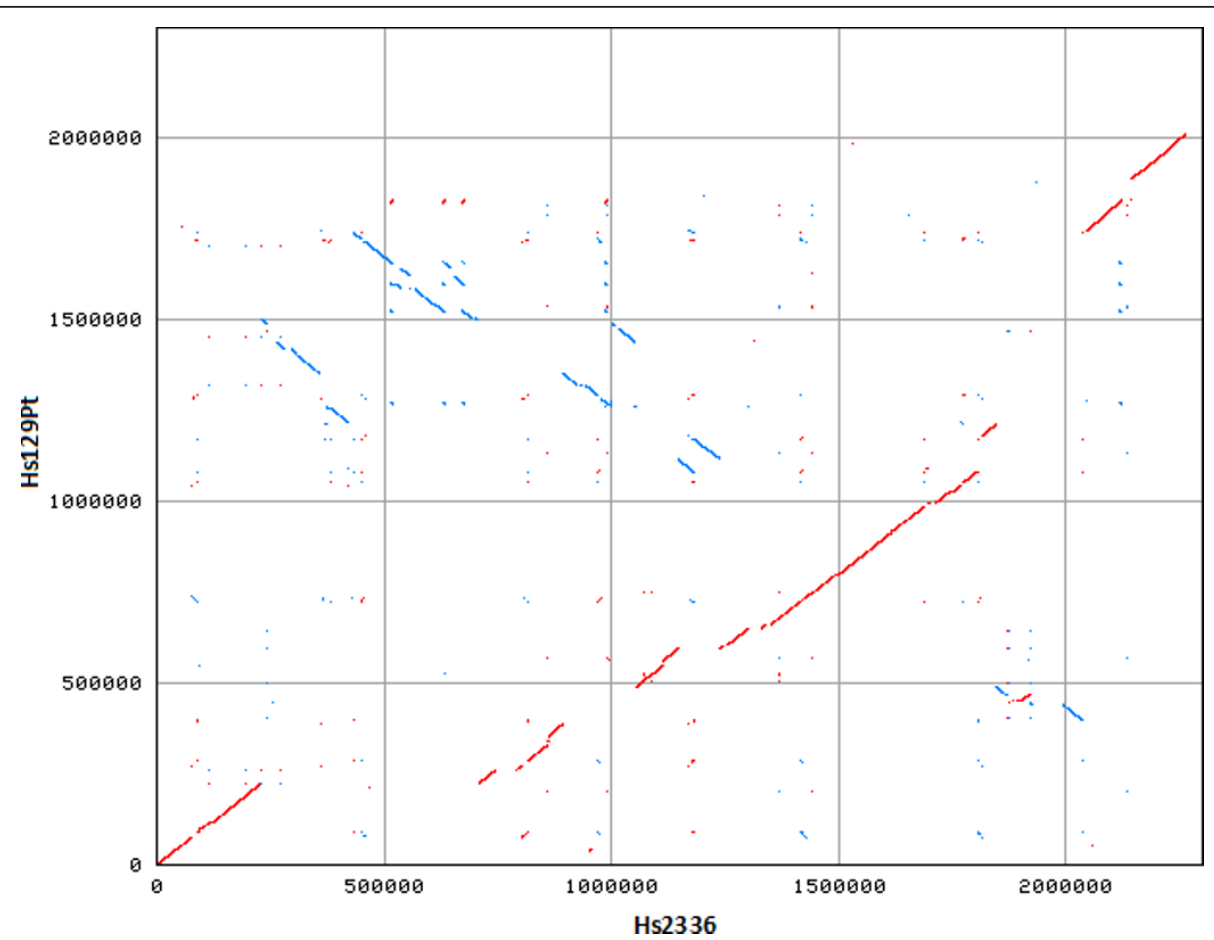

Figure 2 Synteny plot of the chromosomes of strains 2336 and 129Pt generated by NUCmer. Regions of identity between the two chromosomes are shown based on pair-wise alignments. The strain 2336 sequence is represented on the X-axis, and the strain 129Pt sequence is represented on the Y-axis. Numbers indicate nucleotide positions within the respective chromosomes. Plus strand matches are slanted from the bottom left to the upper right corner and are shown in red. Minus strand matches are slanted from the upper left to the lower right and are shown in blue. The number of dots/lines shown in the plot is the same as the number of exact matches found by NUCmer.

least 10 ORFs encoding putative proteins related to bacteriophage structural components. PR IV contained homology to a region of the genome of $H$. influenzae strain 86028NP (9 segments totaling $\sim 4,700$ bp in the $1,707,498 \mathrm{bp}$ to $1,725,687$ bp region, $67-84 \%$ nucleotide identity, $\mathrm{E}$-value $=3 \mathrm{e}-126$ to $1 \mathrm{e}-05)$. Furthermore, the P2 family lysogenic bacteriophage phi-MhaA1-PHL101 from
M. haemolytica serotype A1 contained some of the ORFs found within PR IV of strain 2336.

PR I of strain 129Pt was 6,361 bp shorter than PR III of strain 2336, but the two PRs had a similar GC content. A BLASTN analysis indicated that a sequence of $\sim 20,000 \mathrm{bp}$ was conserved between PR I of strain 129Pt and PR III of strain 2336 (96-99\% nucleotide identity, E-value =0).

Table 2 Characteristics of the prophage regions and genomic islands of H.somni strains 2336 and $129 \mathrm{Pt}$

\begin{tabular}{|c|c|c|c|c|c|}
\hline Strain & Annotation & Chromosomal location & Size (bp) & GC \% & Number of genes \\
\hline \multirow[t]{7}{*}{2336} & PR I & 243787 bp to 255490 bp & 11704 & 40.23 & 13 \\
\hline & $P R \|$ & 759194 bp to $783660 \mathrm{bp}$ & 24467 & 43.95 & 25 \\
\hline & PR III & $1001039 \mathrm{bp}$ to $1049028 \mathrm{bp}$ & 47990 & 39.63 & 55 \\
\hline & PR IV & $1301649 \mathrm{bp}$ to $1329985 \mathrm{bp}$ & 28337 & 39.85 & 39 \\
\hline & Gl I & 1877815 bp to 1902856 bp & 25042 & 37.00 & 21 \\
\hline & GI ॥ & 1963256 bp to 1992078 bp & 28823 & 42.81 & 31 \\
\hline & Gl III & $2124957 \mathrm{bp}$ to $2143800 \mathrm{bp}$ & 18844 & 36.00 & 18 \\
\hline \multirow[t]{7}{*}{$129 \mathrm{Pt}$} & Gl I & $464154 \mathrm{bp}$ to $472854 \mathrm{bp}$ & 8701 & 37.60 & 13 \\
\hline & GI ॥ & 567352 bp to 574742 bp & 7391 & 33.82 & 11 \\
\hline & Gl III & $1524138 \mathrm{bp}$ to $1529684 \mathrm{bp}$ & 5547 & 35.41 & 7 \\
\hline & PR I & $1569138 \mathrm{bp}$ to $1610766 \mathrm{bp}$ & 41629 & 39.37 & 49 \\
\hline & GI IV & $1955573 \mathrm{bp}$ to $1961650 \mathrm{bp}$ & 6078 & 37.86 & 8 \\
\hline & GI V & $1962205 \mathrm{bp}$ to $1966694 \mathrm{bp}$ & 4490 & 31.27 & 7 \\
\hline & $\mathrm{Gl} \mathrm{VI}$ & 1969857 bp to 1974596 bp & 4740 & 38.19 & 9 \\
\hline
\end{tabular}


A two-way comparison indicated that an $11,525 \mathrm{bp}$ segment $(1,006,964$ bp to $1,018,488 \mathrm{bp}, 37.38 \%$ GC) within PR III, and a 1,255 bp segment from the 5' side of PR III of strain 2336 were absent in strain 129Pt (Figure 3A). A similar comparison indicated that a 5,740 bp segment (1,595,978 bp to $1,601,717 \mathrm{bp}, 35.47 \%$ GC) within PR I, and a 2,192 bp segment from the 3' side of PR I, of strain $129 \mathrm{Pt}$ were absent in strain 2336 (Figure 3B). Bacteriophages within the Prophinder database containing some of the predicted ORFs from PRs I-IV of strain 2336 and PR I of strain 129Pt are shown in Figure 4.

Manual curation indicated that strains 2336 and 129Pt contained 3 and 6 GIs, comprising a total of 72,709 bp and 36,947 bp, respectively (Table 2). GI I of strain 2336 contained 17 ORFs of unknown function and a similar sequence was not found in strain $129 \mathrm{Pt}$ or other members of the Pasteurellaceae. GI II of strain 2336 was the longest, had a higher GC content ( 43\%) than the overall genome, and contained 16 ORFs of unknown function. The genome of P. multocida strain Pm70 contained several short sequences that had homology to this region (e.g., 3 segments totaling $5,433 \mathrm{bp}$ in the $2,174,533 \mathrm{bp}$ to $2,187,234$ bp region, $82-99 \%$ nucleotide identity, E-value $=0)$. GI III of strain 2336 contained 4 ORFs encoding putative transposases ([GenBank:HSM_1871, [GenBank:HSM_1874, [GenBank:HSM_1883, and [GenBank:HSM_1887] and 8 ORFs of unknown function. Strain 129Pt lacked an analogous GI, but contained homologs of the genes encoding putative transposases. A 4,640 bp sequence from Escherichia coli strain 49 [GenBank:U23723] had homology to GI III of strain 2336 (72\% identity across 3,270 bp, E-value = $0)$.

GI I of strain 129Pt contained an ORF encoding a resolvase/integrase-like protein [GenBank:HS_0445] and 6 ORFs of unknown function. A similar sequence was not found in strain 2336, but H. parasuis strain SH0165 contained several short sequences with homology to this region (e.g., 3 segments totaling 2,040 bp, 68-79\% nucleotide identity, E-values $=0$ to $1 \mathrm{e}-06)$. GI II of strain $129 \mathrm{Pt}$ contained an ORF encoding a putative phage DNA primase-like protein [GenBank:HS_0533] and 9 ORFs of unknown function. A similar sequence was not found in strain 2336 or other members of the Pasteurellaceae. GI III of strain 129Pt contained ORFs encoding a putative phage terminase protein [GenBank:HS_1334], a prophage regulatory element [GenBank:HS_1335], an integrase [GenBank:HS_1337], and 4 ORFs of unknown function. A similar sequence was not found in strain 2336, but Aggregatibacter aphrophilus strain NJ8700 contained several short sequences that had homology to this region (e.g., 2 segments totaling $1,334 \mathrm{bp}$ in the $1,818,930 \mathrm{bp}$ to $1,821,098$ bp region, 74-80\% nucleotide identity, E-values $=0$ to $4 \mathrm{e}-35)$. All predicted ORFs from GIs IV and V of strain 129Pt were of unknown function and strain 2336 contained several short sequences that had homology to these regions (e.g., 7 segments totaling 2,184 bp in the $1,965,862$ bp to $1,990,890$ bp region of GI II, $67-92 \%$ nucleotide identity, E-values $=9 \mathrm{e}-42$ to $1 \mathrm{e}-10)$. The chromosome of strain 2336 had no regions of homology to GI VI of strain 129Pt, but H. parasuis strain SH0165 contained several short sequences that had homology to this region (e.g., 3 segments totaling 1,573 bp, 68\% nucleotide identity, E-values $=2 \mathrm{e}-51$ to $2 \mathrm{e}-13$ ).

\section{Comparison of insertion sequences}

Insertion sequence finder indicated that strains 2336 and $129 \mathrm{Pt}$ contained several IS elements distributed throughout the chromosomes. Not surprisingly, some of these IS elements were found within the PRs and/or GIs described above. Insertion sequence 1016 (IS1016), consisting of a transposase [217 amino acids (aa)] flanked by $18-29$ bp of terminal inverted repeats, is a member of the IS1595 superfamily [35,36]. Strain 2336 contained 4 fulllength ([GenBank:HSM_0851], [GenBank:HSM_1211], [GenBank:HSM_1267], and [GenBank:HSM_1883]) and three truncated copies of IS1016. GI III of strain 2336 included [GenBank:HSM_1883] and one of the three truncated copies of IS1016. Strain 129Pt contained 5 fulllength copies of IS1016 ([GenBank:HS_0324], [GenBank: HS_0642], [GenBank:HS_1116], [GenBank:HS_1679], and [GenBank:HS_1709]). A gene in strain 129Pt (licA; [GenBank:HS_1461]), encoding a choline kinase that is

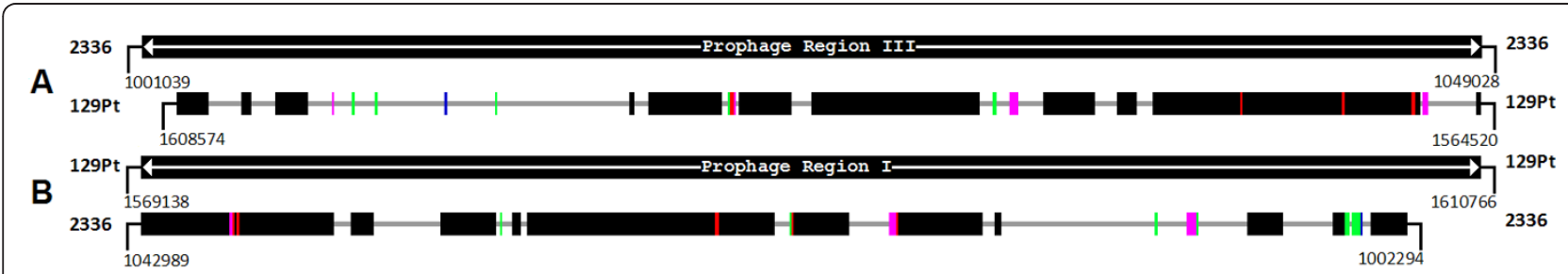

Figure 3 (A) BLASTN of prophage region III of strain 2336 (47,990 bp) against the chromosome of strain 129Pt and (B) BLASTN of prophage region I of strain $129 \mathrm{Pt}(\mathbf{4 1 , 6 2 9} \mathbf{b p})$ against the chromosome of strain $\mathbf{2 3 3 6}$. The numbers below each of the maps refer to the nucleotide positions in the respective chromosomes. The matches are color-coded according to the BLASTN alignment scores (Red $<40$, Blue $=$ 40-50, Green $=50-80$, Pink = 80-200, and Black $\geq 200$; a gray line indicates the absence of a significant match). 


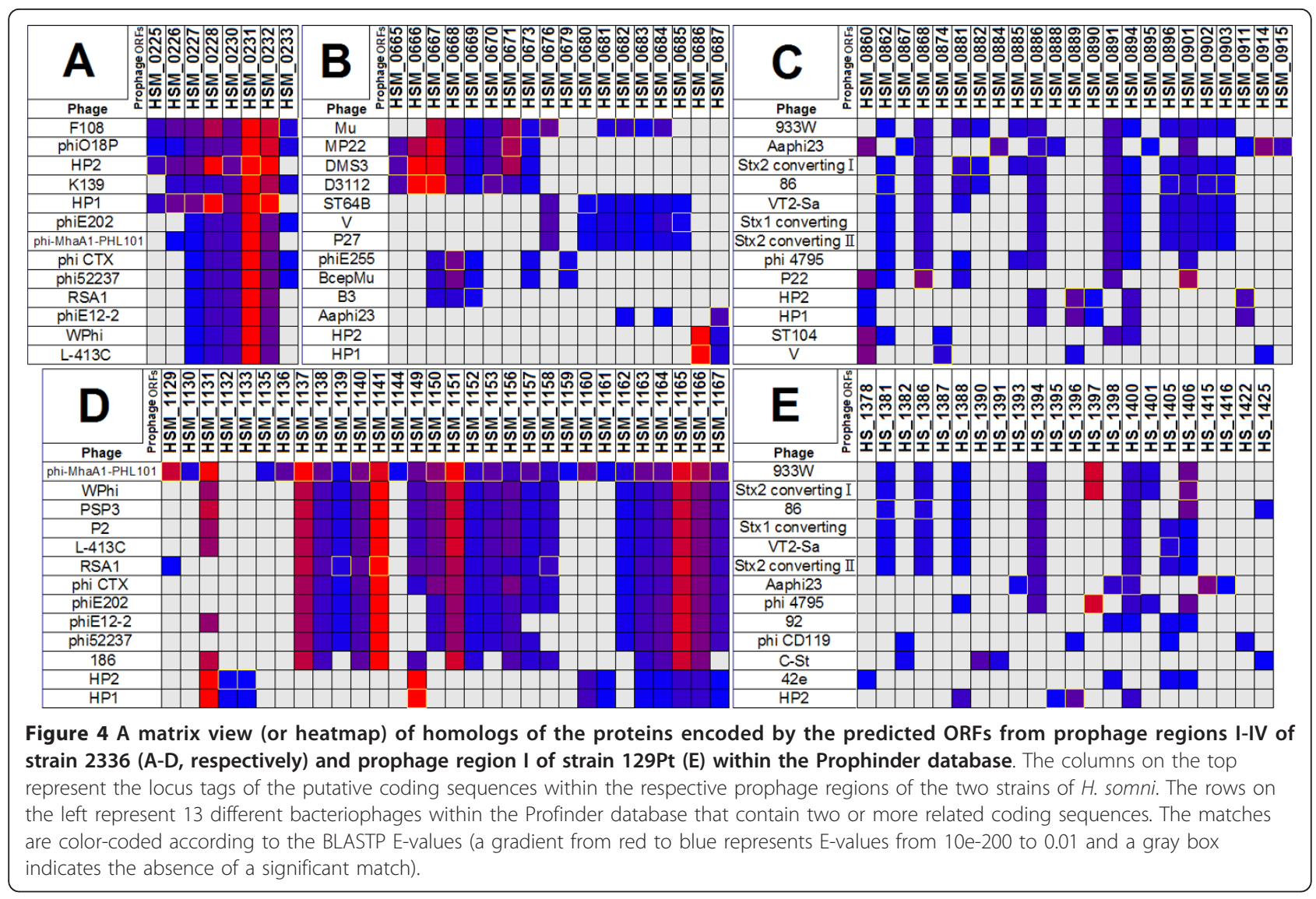

necessary for the synthesis of phosphorylcholine [37], appeared to be interrupted due to an insertion-excision event involving IS1016, since two partial homologs of the transposase gene occurred upstream of licA.

Strain 2336 contained 4 full-length members of the family IS200/IS605 ([GenBank:HSM_0223] was near PR I and [GenBank:HSM_1626] and [GenBank:HSM_1631] were near GI I). Strain 129Pt contained 3 full-length ([GenBank:HS_0486], [GenBank:HS_0583], and [GenBank: HS_1390]) and 6 truncated ([GenBank:HS_0224], [GenBank:HS_0666], [GenBank:HS_0667], [GenBank:HS_0668], [GenBank:HS_0716], and [GenBank:HS_0717]) members of the family IS200/IS605. PR I of strain 129Pt included [GenBank:HS_1390]. Strain 2336 contained a full-length member of the family IS30 ([GenBank:HSM_1680]) and a truncated member of the family IS1182/IS5 ([GenBank: HSM_1887]). Strain 2336 contained a full-length member of the family IS481 ([GenBank:HSM_0451]), whereas strain 129Pt contained a truncated copy of the same ORF ([GenBank:HS_1518]). Strain 2336 also contained truncated members of the family IS3 ([GenBank:HSM_0531] and [GenBank:HSM_0532]). The closest homologs of H. somni IS200/IS605 and IS1595 elements were found in M. haemolytica (e.g., [GenBank:MHA_0340], 92\% identity, E-value
$=0$ ) and H. influenzae (e.g., [GenBank:CGSHiII_08436], $86 \%$ identity, E-value $=0$ ). However, the closest homologs of the IS30 element from strain 2336 were found in $A$. pleuropneumoniae (e.g., [GenBank:APP7_0397], 99\% identity, E-value $=0$ ) and $H$. parasuis (e.g., [GenBank: HAPS_1772], 99\% identity, E-value = 8e-180).

\section{BLAST comparison of protein sets}

Strains 2336 and $129 \mathrm{Pt}$ contained 1550 predicted protein coding genes in common (bidirectional best hits, at least $90 \%$ identity at the predicted protein level). In strain 2336, 440 ORFs could not be assigned a function based on BLAST analysis and were therefore annotated as encoding hypothetical or conserved hypothetical proteins. In strain 129Pt, 429 ORFs were annotated as encoding hypothetical or conserved hypothetical proteins. Among hypothetical proteins that were common to both strains, 30 did not have homologs outside the genus. Pairwise BLAST comparisons indicated that strain 2336 contained 311 putative protein coding genes with no homologs in strain 129Pt (additional file 1). Within this subset, proteins encoded by 302 genes had at least 51 aa and 9 genes ([GenBank:HSM_0528], [GenBank:HSM_0530], 
[GenBank:HSM_0532], [GenBank:HSM_0603], [GenBank:HSM_1185], [GenBank:HSM_1483], [GenBank: HSM_1636], [GenBank:HSM_1742], and [GenBank: HSM_1743]) had 38-50 aa. Strain 129Pt contained 165 putative protein coding genes with no homologs in strain 2336 (additional file 2). Within this subset, proteins encoded by all 165 genes had at least 51 aa.

In both strains, a vast majority of putative HTGs appeared to have had their origins among members of gammaproteobacteria (mostly within Pasteurellales and Enterobacteriales). Putative HTGs with possible origins among members of betaproteobacteria (27 in strain 2336, 11 in strain $129 \mathrm{Pt}$ ) and alphaproteobacteria (1 in strain 2336, 6 in strain 129Pt) were also identified. Among HTGs identified were those encoding proteins putatively involved in virulence (e.g., filamentous hemagglutinins, proteases, and antibiotic resistance regulators). A complete list of these genes is available at the 'Organism Details' sections for strains 2336 and 129Pt within IMG. Other strain-specific genes identified encoded DNA methylases (7 in strain 2336, none in strain 129Pt), transposases (8 in strain 2336, 1 in strain $129 \mathrm{Pt}$ ), ABC transporters (5 in strain 2336, none in strain 129Pt), ATPases (4 in strain 2336, none in strain $129 \mathrm{Pt}$ ), transcriptional regulators (14 in strain 2336, 3 in strain $129 \mathrm{Pt}$ ), kinases (2 in strain 2336, 1 in strain 129Pt), and several proteins related to bacteriophage functions (e.g., of the 10 integrase/resolvase-related genes found in strain $129 \mathrm{Pt}$, six have no homologs in strain 2336 and of the 7 integrase/ resolvase-related genes found in strain 2336, 2 have no homologs in strain 129Pt). Excluding intergenic regions, the total length of sequence that was associated with specific genes in strain 2336 was 254,052 bp ( 11\% of the genome), and was 98,016 bp ( $~ 5 \%$ of the genome) in specific genes of strain 129Pt.

\section{Identification of genes encoding polysaccharide biosynthesis/modification enzymes}

A search of the NCBI non-redundant protein database using the BLASTP algorithm identified 17 ORFs that encode putative glycosyltransferases (GTs) in the genomes of strains 2336 and 129Pt. Seven of these ORFs were common to both genomes (at least 96\% identity at the predicted protein level), 8 were found only in strain 2336, and 2 were found only in strain 129Pt. Among the ORFs encoding putative GTs common to both strains, 5 contained simple sequence repeats (SSRs), and 4 of the 8 ORFs encoding GTs found in strain 2336 contained SSRs. A list of putative GTs and their SSRs identified in both strains are shown in Table 3. Among the ORFs that encode putative GTs in $H$. somni strains, three ([GenBank:HSM_0148/HS_0275], [GenBank:HSM_0856], and [GenBank:HSM_1552/HS_1067]) had no homologs among other members of the Pasteurellaceae and six
([GenBank:HS_0636], [GenBank:HSM_0975], [GenBank: HSM_0977], [GenBank:HSM_0978], [GenBank:HSM_ 1426], and [GenBank:HSM_1794]) had distant homologs ( $<50 \%$ identity) among other members of the Pasteurellaceae (Table 3). These ORFs were annotated based on their location on the chromosome (e.g., the [GenBank: HSM_0975-0979] cluster), comparative analyses using the Swiss-Prot database (e.g., [GenBank:HSM_0148], [GenBank:HSM_0856], and [GenBank:HSM_1552]), and their homology to genes encoding putative GTs in nonPasteurellaceae genomes (e.g., [GenBank:HS_0636], [GenBank:HSM_1426], and [GenBank:HSM_1794]).

The lipooligosaccharide biosynthesis $(l o b)$ gene cluster consisting of $l o b 1$ and $l o b 2 \bar{A} B C D$ ORFs encoding glycosyltransferases involved in attaching the outer core glycoses of the LOS was previously identified in strain 738, which is an LOS phase variant of strain 2336 [5,7]. Strain $129 \mathrm{Pt}$ encoded full-length homologs of $l o b 1$ and $l o b 2 D$, but only the 5' ends of $l o b 2 A$ and $l o b 2 C$ and lacked $l o b 2 B$ [13]. Strain 2336 contained full-length homologs of lob1 and lob $2 A B C$, but a truncated homolog of lob2D (table 3 ). The variations in the lob loci in the genomes of strains 2336 and $129 \mathrm{Pt}$ correlate with the differences in the structures of the LOS of these strains, as determined by NMR spectroscopy and mass spectrometry [16,38]. In addition, both strains contained ORFs encoding a phosphoheptose isomerase ([GenBank:HSM_0840] and [GenBank:HS_1238], gmhA), D,D-heptose 1,7-bisphosphate phosphatase ([GenBank:HSM_0572] and [GenBank:HS_1532], $g m h B)$, ADPL-glycero-D-mannoheptose-6-epimerase ([GenBank: HSM_0397] and [GenBank:HS_1613], rfaD), bifunctional heptose 7-phosphate kinase/heptose 1-phosphate adenyltransferase ([GenBank:HSM_0925] and [GenBank: HS_0576, rfaE), and bifunctional $N$-acetylglucosamine-1phosphate uridyltransferase/glucosamine-1-phosphate acetyltransferase ([GenBank:HSM_0204] and [GenBank: HS_0333], $g l m U)$. These five genes/enzymes were predicted to be involved in LOS core biosynthesis in strains 2336 and $129 \mathrm{Pt}$.

Strain 2336 also contained a locus that encodes proteins putatively involved in exopolysaccharide (EPS) and/or LOS biosynthesis ([GenBank:HSM_1061] or $\operatorname{csr} A$, carbon storage regulator; [GenBank:HSM_1062] or manB phosphomannomutase; [GenBank:HSM_1063] or galU, UTPglucose-1-phosphate uridylyltransferase). Strain $129 \mathrm{Pt}$ contained a similar locus ([GenBank:HS_1117] to [GenBank:HS_1119]) that had an IS1016 element ([GenBank: HS_1116], transposase) adjacent to it. Furthermore, both strains contained ORFs encoding a putative UDP-glucose 4-epimerase ([GenBank:HSM_1256] and [GenBank:HS_ 0789], galE), phosphoglucomutase ([GenBank:HSM_1832] and [GenBank:HS_1670], pgmB), and dTDP-glucose 4,6dehydratase ([GenBank:HSM_1118] and [GenBank:HS 
Table 3 Putative glycosyltransferase genes of H.somni strains

\begin{tabular}{|c|c|c|c|c|}
\hline $\begin{array}{l}\text { Strain } 2336 \text { locus tag, } \\
\text { protein, }{ }^{1}(\text { SSR, if present) } n\end{array}$ & $\begin{array}{l}\text { Strain } 129 \mathrm{Pt} \text { locus tag, } \\
\text { protein, }{ }^{1}(\mathrm{SSR}, \text { if present) } \mathrm{n}\end{array}$ & $\begin{array}{l}\text { SWISS-PROT } \\
\text { accession number }\end{array}$ & $\begin{array}{l}\text { Closest Pasteurellaceae homolog Bacterium, } \\
\text { locus tag, protein size, }{ }^{2} \text { identity }\end{array}$ & $\begin{array}{l}\text { Closest GT homolog (Outside Pasteurellaceae) } \\
\text { locus tag, protein, }{ }^{2} \text { identity }\end{array}$ \\
\hline $\begin{array}{l}\text { [GenBank:HSM_0148], } 260 \text { aa, } \\
(G)_{9}\end{array}$ & [GenBank:HS_0275], 259 aa, (G)6 & $\begin{array}{l}\text { [Swiss-Prot:B0UVK9/ } \\
\text { Q011L8] }\end{array}$ & None & $\begin{array}{l}\text { Acinetobacter johnsonii, [GenBank:EEY94992], } 281 \text { aa, } \\
44 \%\end{array}$ \\
\hline $\begin{array}{l}\text { [GenBank:HSM_0164], } 522 \text { aa, } \\
(\mathrm{G})_{9}\end{array}$ & None & [Swiss-Prot:B0UVM5] & $\begin{array}{l}\text { Actinobacillus succinogenes, [GenBank:Asuc_0521], } \\
522 \text { aa, } 63\end{array}$ & Neisseria mucosa, [GenBank:EFC88975], 517 aa, 53\% \\
\hline${ }^{3}$ [GenBank:HSM_0398], 346 аa & [GenBank:HS_1612], 346 aa & $\begin{array}{l}\text { [Swiss-Prot } \\
\text { BOUWU4/Q01570] }\end{array}$ & $\begin{array}{l}\text { Mannheimia, succiniciproducens [GenBank:MS2260], } \\
346 \mathrm{aa}, 78 \%\end{array}$ & $\begin{array}{l}\text { Yersinia pseudotuberculosis, [GenBank:YPTB0054], } 354 \\
\text { aa, } 64 \%\end{array}$ \\
\hline${ }^{3}$ [GenBank:HSM_0399], 321 aа & [GenBank:HS_1611], 321 aa & $\begin{array}{l}\text { [Swiss-Prot BOUWU5/ } \\
\text { Q01571] }\end{array}$ & Mannheimia succiniciproducens & $\begin{array}{l}\text { Providencia rettgeri, [GenBank:EFE51471], [GenBank: } \\
\text { MS2259], } 321 \mathrm{aa}, 67 \% 322 \mathrm{aa}, 63 \%\end{array}$ \\
\hline${ }^{4}[$ GenBank:HSM_0856], 314 aa & None & [Swiss-Prot:BOUST9] & None & Rhodopirellula baltica, [GenBank:RB9243], 326 aa, 35\% \\
\hline $\begin{array}{l}{ }^{5}[\text { GenBank:HSM_0975a], } 157 \text { aa, } \\
(\text { CAGT) } 18\end{array}$ & $\begin{array}{l}{ }^{5}[\text { GenBank:HS_0636], } 354 \text { aa, } \\
(\text { CAGT) } 29\end{array}$ & [Swiss-Prot:Q0I2Z7] & $\begin{array}{l}\text { Haemophilus influenzae, [GenBank:NTHI0365], } 312 \\
\text { aa, 38\% }\end{array}$ & $\begin{array}{l}\text { Neisseria meningitidis, [GenBank:AF355193_3], } 311 \text { aa, } \\
\text { 35\% }\end{array}$ \\
\hline $\begin{array}{l}\text { [GenBank:HSM_0975], } 231 \text { aa, } \\
(\mathrm{AGA})_{3}\end{array}$ & None & [Swiss-Prot:B0UT56] & $\begin{array}{l}\text { Pasteurella dagmatis, [GenBank:EEX51329], } 334 \text { aa, } \\
40 \%\end{array}$ & Neisseria lactamica, [GenBank:AAN08512], 251aa, 32\% \\
\hline${ }^{6}[$ GenBank:HSM_0976], 63 aа & [GenBank:HS_0636a] & [Swiss-Prot:BOUT57] & $\begin{array}{l}\text { Pasteurella multocida, [GenBank:PM1140], } 337 \text { aa, } \\
61 \%\end{array}$ & $\begin{array}{l}\text { Streptococcus parasanguinis, [GenBank:ACF35266], } \\
297 \text { aa, 59\% }\end{array}$ \\
\hline $\begin{array}{l}{ }^{7}[\text { GenBank:HSM_0977], } 277 \text { aa, } \\
(\text { GAGA) })_{11}\end{array}$ & None & [Swiss-Prot:B0UT58] & $\begin{array}{l}\text { Aggregatibacter actinomycetemcomitans GenBank: } \\
\text { D11S_0429], } 282 \text { aa, } 43 \%\end{array}$ & $\begin{array}{l}\text { Neisseria meningitidis, [GenBank:NMC1901], } 275 \text { aa, } \\
41 \%\end{array}$ \\
\hline $\begin{array}{l}{ }^{8}[\text { GenBank:HSM_0978], } 271 \text { aa, } \\
(\mathrm{GA})_{20}\end{array}$ & [GenBank:HS_0637], 158 aа & [Swiss-Prot:BOUT59] & $\begin{array}{l}\text { Aggregatibacter actinomycetemcomitans [GenBank: } \\
\text { D11S_0429], } 282 \text { aa, 43\% }\end{array}$ & $\begin{array}{l}\text { Neisseria meningitidis, [GenBank:CAX50883], } 280 \text { aa, } \\
39 \%\end{array}$ \\
\hline $\begin{array}{l}{ }^{9}[\text { GenBank:HSM_0979], } 293 \text { aа, } \\
\left(\text { (CAAT) }{ }_{33}\right.\end{array}$ & $\begin{array}{l}\text { [GenBank:HS_0638], } 297 \text { aa, } \\
(\text { CAAT) } 36\end{array}$ & $\begin{array}{l}\text { [Swiss-Prot:BOUT60/ } \\
\text { Q012Z5] }\end{array}$ & $\begin{array}{l}\text { Actinobacillus succinogenes, [GenBank:Asuc_0526], } \\
265 \text { aa, 52\% }\end{array}$ & $\begin{array}{l}\text { Photobacterium profundum, [GenBank:PBPRA0217], } \\
253 \mathrm{aa}, 41 \%\end{array}$ \\
\hline [GenBank:HSM_1426], 300 aa & None & [Swiss-Prot:BOUUE7] & $\begin{array}{l}\text { Haemophilus influenzae, [GenBank:HI0871], } 306 \text { aa, } \\
42 \%\end{array}$ & $\begin{array}{l}\text { Fusobacterium nucleatum, [GenBank:EDK88896], } 326 \\
\text { aa, 38\% }\end{array}$ \\
\hline $\begin{array}{l}\text { [GenBank:HSM_1552], } 196 \text { aa, } \\
(G)_{9}\end{array}$ & [GenBank:HS_1067], 194 aa, (G)6 & $\begin{array}{l}\text { [Swiss-Prot:BOUUS9/ } \\
\text { Q01479] }\end{array}$ & None & $\begin{array}{l}\text { Edwardsiella ictaluri, [GenBank:NT01EI_2547], } 247 \text { aa, } \\
27 \%\end{array}$ \\
\hline${ }^{10}$ [GenBank:HSM_1794], 311 aа & None & [Swiss-Prot:BOUWB1] & $\begin{array}{l}\text { Mannheimia haemolytica, [GenBank:MHA_0104], } \\
312 \text { aa, } 47 \%\end{array}$ & $\begin{array}{l}\text { Streptococcus agalactiae [GenBank:AAR29926], } 299 \text { aa, } \\
\text { 39\% }\end{array}$ \\
\hline $\begin{array}{l}\text { [GenBank:HSM_2001], } 242 \text { aa, } \\
(\mathrm{GGT})_{3}\end{array}$ & $\begin{array}{l}\text { [GenBank:HS_0116], } 242 \text { aa, } \\
(\mathrm{GGT})_{3}\end{array}$ & $\begin{array}{l}\text { [Swiss-Prot:BOURS6/ } \\
\text { Q01108] }\end{array}$ & $\begin{array}{l}\text { Aggregatibacter aphrophilus, [GenBank: } \\
\text { NT05HA_1706], } 240 \text { aa, } 61 \%\end{array}$ & [GenBank:EER56028], 241 aа, 55\% \\
\hline None & [GenBank:HS_0291], 256 aа & [Swiss-Prot:Q011K2] & $\begin{array}{l}\text { Mannheimia succiniciproducens [GenBank:MS0438], } \\
253 \mathrm{aa}, 76 \%\end{array}$ & Vibrio shilonii [GenBank:EDL53925], 259 aa, 62\% \\
\hline None & [GenBank:HS_0292], 348 aa & [Swiss-Prot:Q011K1] & $\begin{array}{l}\text { Actinobacillus minor NM305, [GenBank:EER47697], } \\
340 \text { aa, 57\% }\end{array}$ & $\begin{array}{l}\text { Vibrio parahaemolyticus, [GenBank:EED27646], } 341 \text { aa, } \\
39 \%\end{array}$ \\
\hline
\end{tabular}

Simple sequence repeats identified within the predicted ORF.

${ }^{2}$ Identity at the predicted protein level as compared to strain 2336 or strain $129 \mathrm{Pt}$ full-length or partial homolog.

HSM_0398 and 0399 are predicted to encode lipopolysaccharide heptosyltransferases II and I, respectively.

${ }^{4} \mathrm{HSM} 0856$ contains 28 copies of CAGT 67 bp upstream of the predicted start codon.

${ }^{5} \mathrm{HS} \_0636$ (lob2D), encoding a putative glycosyl transferase [13], is truncated in strain 2336 (HSM_0975a).

${ }^{6} \mathrm{HSM} \_0976$ (lob2C), encoding a putative glycosyltransferase-like protein, has an 117 bp homolog in strain 129Pt (HS_0636a).

${ }^{7} \mathrm{HSM} \_0977$ (lob2B) has been predicted to encode an $\mathrm{N}$-acetylglucosamine transferase [5].

${ }^{8} \mathrm{HSM} 0978$ (lob2A), encoding a putative glycosyl transferase, had 66\% identity to HSM 0977 (Iob2B) at the predicted protein level.

${ }^{9} \mathrm{HSM}$ 0979/HS_0638 (lob1) has been predicted to encode a galactosyl transferase [7].

${ }^{10}$ HSM_1794 contains 4 copies of CAAGCCTACAAGCCTACAAGCCTA 262 bp upstream of the predicted start codon. 
0707], $r m l B)$. These three genes/enzymes were predicted to be involved in polysaccharide biosynthesis/modification.

\section{Acquisition and loss of genes encoding filamentous hemagglutinins}

The chromosome of $H$. somni strain 2336 contained four loci that have twelve putative genes encoding proteins homologous to $\mathrm{FhaB}$, and four putative genes encoding proteins homologous to FhaC. Locus I of strain 2336 was 17,222 bp (GC content of $40 \%$ ) and contained genes encoding four FhaB homologs ([GenBank:HSM_0268], [GenBank:HSM_0270], [GenBank: HSM_0272], and [GenBank:HSM_0274]) and one FhaC homolog ([GenBank:HSM_0267]). No transposon or phage sequences were present in this locus. In contrast, strain $129 \mathrm{Pt}$ had a locus containing genes that flanked locus I of strain 2336, but did not contain the FhaB and FhaC homologs (Figure 5, locus I). Locus II of strain 2336 was 6,712 bp (GC content of 26\%) and contained genes encoding FhaB ([GenBank:HSM_1090]) and FhaC ([GenBank:HSM_1089]), which appeared to be associated with a transposon. Strain 129Pt had a locus containing genes that flanked locus II of strain 2336, but did not contain the FhaB and FhaC homologs (Figure 5, locus II).

Locus III of strain 2336 was 14,066 bp (GC content of 37\%) and contained genes encoding FhaB ([GenBank: HSM_1489]) and FhaC ([GenBank:HSM_1490]). The fhaB $(12,288 \mathrm{bp})$ of this locus was the second largest gene in the genome and the largest among the 12 homologs. This gene encoded a putative protein homologous to the high molecular weight immunoglobulin-binding protein of $H$. somni and the large supernatant proteins (Lsp1 and Lsp2) of H. ducreyi that have been previously described [39]. No transposon or phage regions were apparent in this locus. Strain 129Pt had a locus containing genes that flank locus III of strain 2336, but did not contain the FhaB and FhaC homologs (Figure 5, locus III). Locus IV of strain 2336 was 21,841 bp (GC content of $38 \%$ ) and contained genes encoding six FhaB homologs ([GenBank:HSM_1638], [GenBank:HSM_1641], [GenBank:HSM_1643], [GenBank:HSM_1646], [GenBank:HSM_1647], [GenBank:HSM_1651]). A truncated gene encoding FhaC ([GenBank:HSM_1653], 98 aa) was also found in this locus. A partial homolog of [GenBank: HSM_1647] was found in strain 129Pt ([GenBank: HS_0540], $153 \mathrm{aa}$ ), which appeared to be the only region in the chromosome of strain $129 \mathrm{Pt}$ with a sequence related to the fhaB genes (Figure 5, locus IV).

The twelve putative FhaB homologs found in the four loci of strain 2336 varied in size, with the smallest and largest being 83 aa and 4,095 aa, respectively. Phylogenetic comparison indicated that the four FhaB homologs within locus I were most closely related to each other as were the six FhaB homologs within locus IV (data not shown). The FhaC homologs of loci I (581 aa) and III (586 aa) were more closely related to each other than to FhaC from locus II (450 aa). Multiple sequence alignment of N-terminal fragments of FhaB homologs from the four loci of strain 2336 with those of Bordetella pertussis FHA and Proteus mirabilis HpmA showed that they contain several common features (Figure 6). Of the many residues that are shown to be involved in B. pertussis FHA secretion, 6 (four asparagine and one each of serine and glutamic acid) were conserved in all six homologs and 2 (an asparagine and a methionine) were conserved in five of the homologs (Figure 6). However, the NPNG (Figure 6, S2) and CXXC (Figure 6, S3) motifs that may play a role in stabilization of the helical structure were conserved in only three homologs.

\section{Acquisition of a gene encoding subtilisin-like protease}

H. somni strain 2336 GI III contained a gene encoding a putative subtilisin-like serine protease ([GenBank: HSM_1889], 730 aa; Figure 5, locus V, arrow (gene) 10). A BLASTP search revealed that the non-redundant GenBank database contained several proteins that were homologous to [GenBank:HSM_1889], with the closest relative being an E. coli protein ([GenBank:AAA64865], 75\% identity, Evalue $=0)$. The NCBI protein clusters database lists [GenBank:HSM_1889] as one of the 16 members of the [GenBank:CLSK923564] cluster (peptidase S8 and S53, subtilisin, kexin, sedolisin). Among the proteins of this cluster, homologs from Ralstonia eutropha JMP134 ([GenBank:Reut_C6419], 45\% identity, E-value = 1e-178), Pseudomonas fluorescens Pf0-1 ([GenBank:Pfl01_5697], 35\% identity, E-value $=2 \mathrm{e}-116)$, and Pseudomonas syringae pv. phaseolicola 1448A ([GenBank:PSPPH_0180], 35\% identity, E-value $=1 \mathrm{e}-115)$ are listed in the prokaryotic subtilase database as subtilases of the D-H-S family.

Genomic comparison of members of the [GenBank: CLSK923564] cluster revealed that in 3 cases (Arthrobacter aurescens TC1, R. eutropha JMP134, and Xanthomonas campestris pv. vesicatoria str. 85-10), the ORF encoding subtilisin was found on plasmids. In the case of Delftia acidovorans SPH-1, Burkholderia ambifaria AMMD, Polaromonas naphthalenivorans CJ2, Chelativorans sp. BNC1, and E. coli O127:H6 str. E2348/69, the ORF encoding subtilisin was found within a prophage region. Furthermore, in the case of Photorhabdus luminescens subsp. laumondii TTO1, P. syringae pv. phaseolicola 1448A, P. fluorescens Pf0-1, Verminephrobacter eiseniae EF01-2, and Xanthomonas oryzae pv. oryzae PXO99A, the ORF encoding subtilisin appeared to be associated with genes encoding transposases. However, in Chromohalobacter salexigens DSM 3043 and Anaeromyxobacter sp. K, the ORF encoding subtilisin was not associated with prophage or transposase sequences. Interestingly, in 15 host species the 


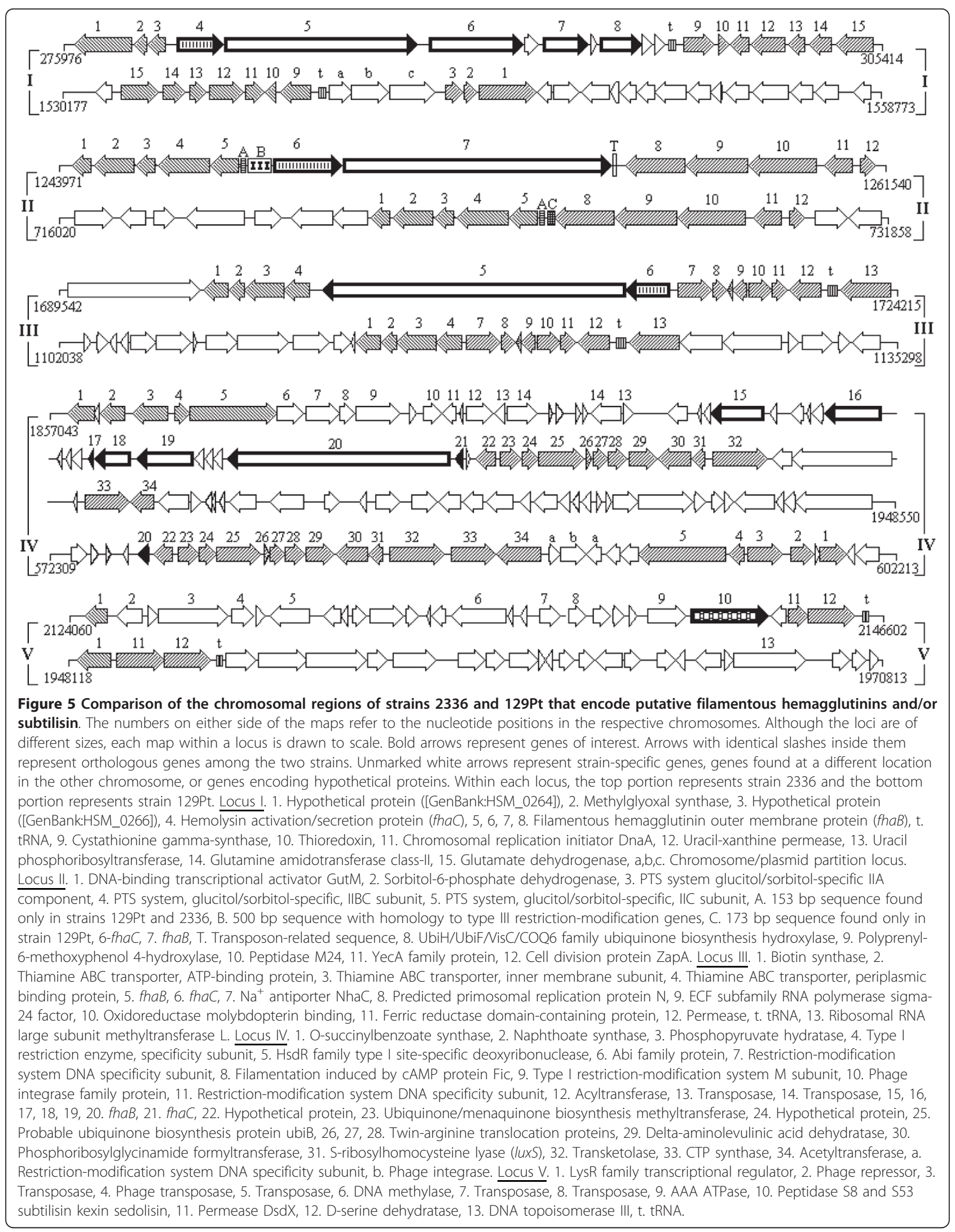




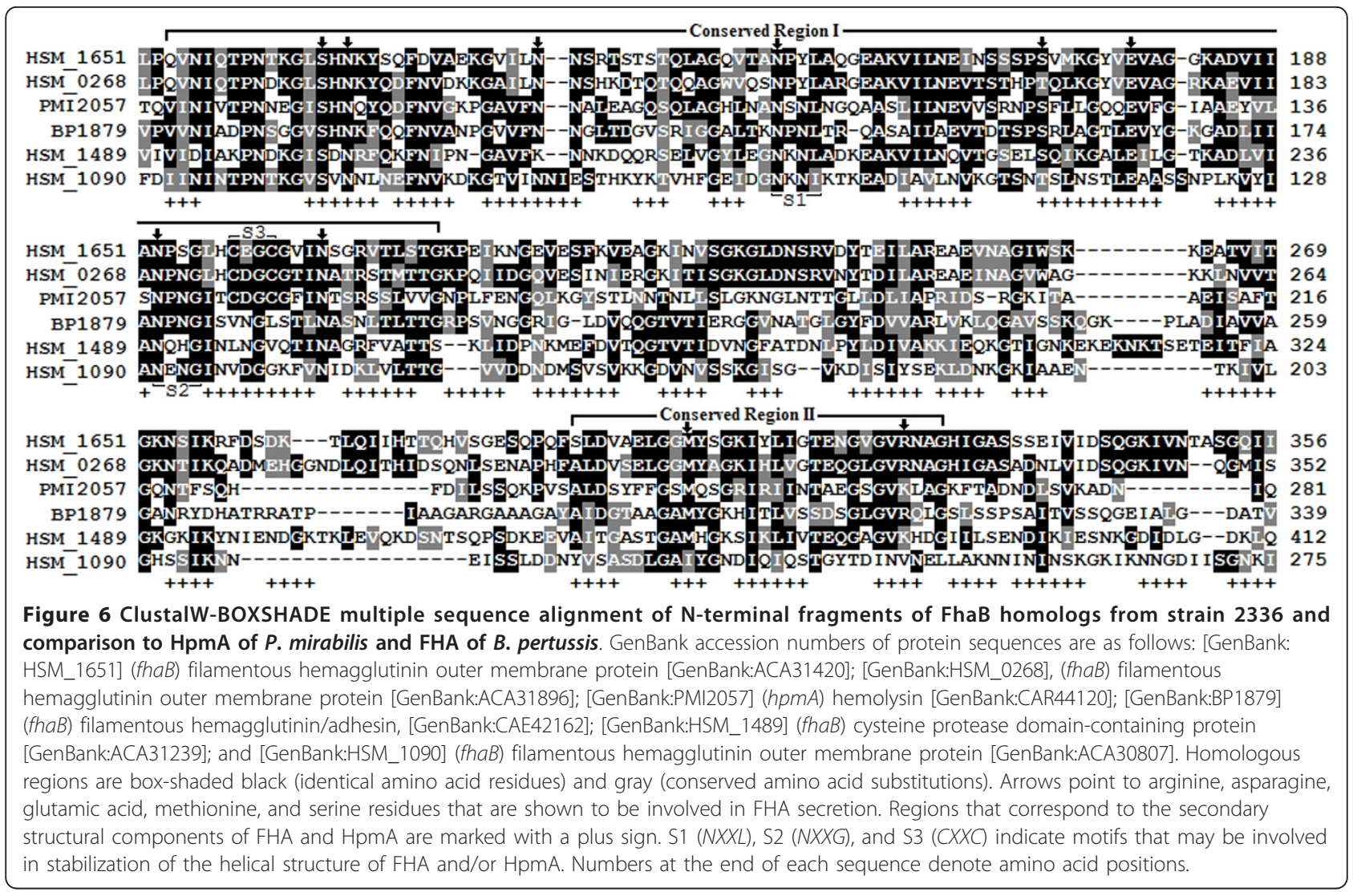

subtilisin ORF formed an operon with an ORF encoding homologous ATPases of the AAA family. In the case of $A$. aurescens $\mathrm{TC1}$, a transposon insertion appears to have disrupted the AAA ATPase-subtilisin operon. The two ORFs have a 4 bp overlap in 8 species and appear to be co-transcribed (Figure 7, left side). Furthermore, comparison of subtilisin sequences from these 8 species indicated that motifs containing the catalytic triad (Asp-His-Ser) were conserved (Figure 7, right side).

\section{Comparison of genes encoding transferrin-binding proteins}

A comparison of the genomes of H. somni strains 2336 and $129 \mathrm{Pt}$ revealed that both strains contained genes

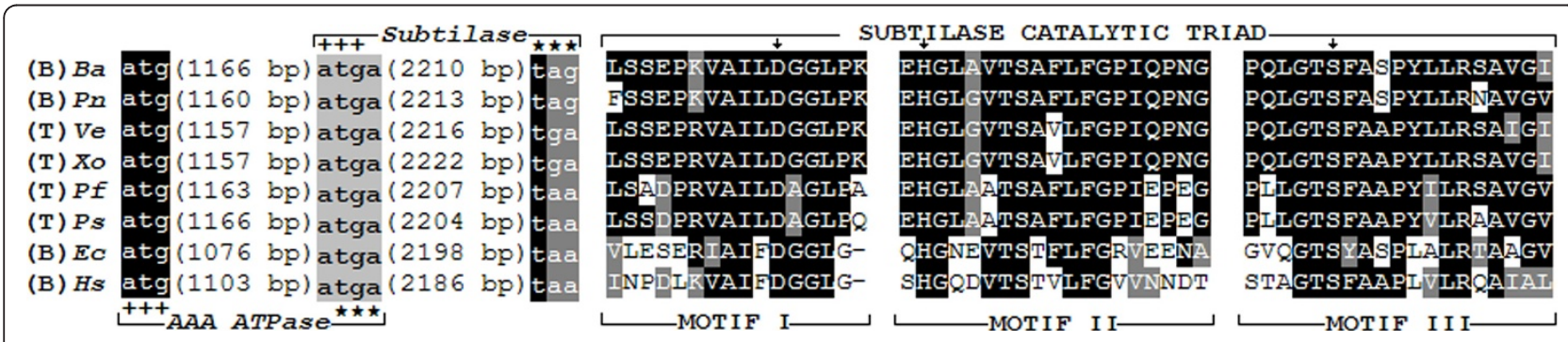

Figure 7 Comparison of 8 members of the NCBI protein cluster CLSK923564. Ba; Burkholderia ambifaria AMMD [GenBank:YP_773860], Pn; Polaromonas naphthalenivorans CJ2 [GenBank:YP_982671], Ve; Verminephrobacter eiseniae EF01-2 [GenBank:YP_998470], Xo; Xanthomonas oryzae pv. oryzae PXO99A [GenBank:YP_001913386], Pf; P. fluorescens Pf0-1 [GenBank:YP_351424], Ps; P. syringae pv. phaseolicola 1448A [GenBank: YP_272486], EC; E. coli O127:H6 str. E2348/69 [GenBank:YP_002329331], Hs; H. somni strain 2336 [GenBank:YP_001785203]. Gene features are shown on the left side: Start and stop codons of ORFs encoding ATPase and sutbilase are marked with plus and asterisk signs, respectively. The length of each ORF is measured from the first base after the start codon to the last base before the stop codon. The 4 bp gene overlap is boxshaded light gray. (B) denotes genes found within prophage-like sequences and (T) denotes genes associated with transposons. ClustalWBOXSHADE multiple sequence alignment of subtilase motifs containing the catalytic triad is shown on the right side: Homologous regions are box-shaded black (identical amino acid residues) and gray (conserved amino acid substitutions). Arrows point to the conserved residues Asp-HisSer of subtilases of the D-H-S family. 
encoding TbpA ([GenBank:HS_0449] and [GenBank: HSM_0750]) and TbpB ([GenBank:HS_0448] and [GenBank:HSM_0749]). In strain 129Pt, GI I was identified immediately upstream of the $t b p$ locus. Furthermore, homologs of H. somni strain 649 TbpA2 were present in strain 129Pt ([GenBank:HS_0582], heme uptake protein) and strain 2336 ([GenBank:HSM_0931], TonB-dependent receptor; [GenBank:HSM_0932], TonB-dependent receptor plug; and [GenBank:HSM_1988], TonB-dependent lactoferrin and transferrin receptor). Pairwise BLAST analysis identified several other putative genes encoding proteins that may also be involved in iron transport. In strain 129Pt these included [GenBank: HS_0069], iron-regulated outer membrane protein; [GenBank:HS_0181], TonB-dependent outer membrane receptor; [GenBank:HS_0728], hemin receptor outer membrane protein; and [GenBank:HS_1306], iron-regulated outer membrane protein. In strain 2336 they included [GenBank:HSM_0047], TonB-dependent receptor; [GenBank:HSM_1168], TonB-dependent hemoglobin/transferrin/lactoferrin family receptor; [GenBank: HSM_1176], outer membrane hemin receptor protein, and [GenBank:HSM_1962], TonB-dependent receptor. Two of the genes, [GenBank:HS_1306] and [GenBank: HSM_1168], were strain-specific. Among others, HS_0582 was associated with a transposase ([GenBank: HS_0583]) and [GenBank:HSM_1168] was found near PR IV.

\section{Overabundance of genes encoding adhesin-like proteins}

Strain 129Pt contained sixteen genes randomly distributed throughout the chromosome ([GenBank:HS_0209], [GenBank:HS_0383], [GenBank:HS_0478], [GenBank: HS_0589], [GenBank:HS_0602], [GenBank:HS_0790], [GenBank:HS_1058], [GenBank:HS_1085], [GenBank: HS_1154], [GenBank:HS_1185], [GenBank:HS_1234], [GenBank:HS_1512], [GenBank:HS_1543], [GenBank: HS_1563], [GenBank:HS_1616], and [GenBank: HS_1632]) with homology to genes that encode proteins of the Yersinia adhesin (YadA) superfamily [13]. Strain 2336 also contained sixteen genes randomly distributed throughout the chromosome ([GenBank:HSM_0077], [GenBank:HSM_0338], [GenBank:HSM_0346], [GenBank:HSM_0377], [GenBank:HSM_0394], [GenBank: HSM_0708], [GenBank:HSM_0844], [GenBank:HSM_09 38], [GenBank:HSM_0953], [GenBank:HSM_1022], [GenBank:HSM_1212], [GenBank:HSM_1257], [GenBank:HSM_1484], [GenBank:HSM_1542], [GenBank: HSM_1571], [GenBank:HSM_1793]) with homology to genes that encode proteins of the YadA superfamily. Adhesin-encoding genes [GenBank:HS_0209] (15,431 bp) and [GenBank:HSM_1257] (13,970 bp) were the largest among all protein coding genes predicted in the chromosomes of strains 129Pt and 2336, respectively.
Within this gene repertoire, [GenBank:HSM_0938] (388 aa) and [GenBank:HS_0589] (386 aa) were $85 \%$ similar to each other at the predicted protein level (75\% identity, Score $=535$ bits, E-value $=1 \mathrm{e}-156$ ) and were associated with genes encoding putative $\mathrm{ABC}$ transporters ([GenBank:HSM_0939-0943] and [GenBank:HS_05900594], respectively, $99 \%$ identity, E-value $=0$ to $5 \mathrm{e}-151$ ). In addition, [GenBank:HSM_0077] (4063 aa) and [GenBank:HS_0209] (5143 aa) were 65\% similar to each other at the predicted protein level (53\% identity, Score $=1009$ bits, E-value $=0$ ) and were also associated with genes encoding putative $\mathrm{ABC}$ transporters ([GenBank: HSM_0079-0081] and [GenBank:HS_0210-0212], respectively, 99\% identity, E-value $=2 \mathrm{e}-146$ to $3 \mathrm{e}-132$ ). Furthermore, although homologs of the $H$. influenzae fimbrial gene cluster (hif $A B C D E$ ) were absent in both strains of $H$. somni, homologs of type IV pili genes (pilABCD) occurred in strains 2336 and 129Pt (e.g., [GenBank:HSM_0123] and [GenBank:HS_0250], [GenBank:HSM_0217] and [GenBank:HS_1430, [GenBank: HSM_0755 and [GenBank:HS_0457], and [GenBank: HSM_0756] and [GenBank:HS_0458], 40-69\% identity, E-values $=1 \mathrm{e}-168$ to $1 \mathrm{e}-38)$. In addition, both strains contained a gene that encoded a putative pseudopilin (pulG, [GenBank:HS_0264] and [GenBank:HSM_0137], $54 \%$ identity to NTHI1109, E-value $=9 \mathrm{e}-51$ ) that may facilitate type II secretion.

\section{Identification of genes encoding transcriptional regulators and drug/metal resistance}

Pairwise BLAST comparisons indicated that strain 2336 contained 14 genes encoding transcriptional regulators with no homologs in strain 129Pt. These included two regulators each that belong to the TetR ([GenBank: HSM_1191] and [GenBank:HSM_1734]) and MerR ([GenBank:HSM_1728] and [GenBank:HSM_1741]) families, one each that belonged to the LysR ([GenBank: HSM_0806]), OmpR ([GenBank:HSM_0817]), and MarR ([GenBank:HSM_1737]) families, and a member of an unassigned family ([GenBank:HSM_0489]; [GenBank: COG2865K]). Among these, members of the TetR, MerR, and MarR families were found within GI II. Strain 2336 also contained a gene adjacent to tet $R$ encoding a tetracycline resistance antiporter (tetH; [GenBank:HSM_1735]). The NCBI protein clusters database lists [GenBank: HSM_1734] as a member of the PRK13756 cluster (TetR; tetracycline repressor of Leuconostoc citreum[GenBank: COG1309K]). The closest homologs of [GenBank: HSM_1734] and [GenBank:HSM_1735] were found in P. multocida (e.g., [GenBank:AAC43249] and [GenBank: AAC43250] encoding a repressor protein and a tetracycline resistance protein, respectively, 99\% identity, E-values $=0$ to $1 \mathrm{e}-116)$. In addition to [GenBank:HSM_1737], strain 2336 also contained [GenBank:HSM_1736] 
(encoding small multidrug resistance protein). [GenBank: HSM_1191] is listed as one of the members of the [GenBank:CLSK391246] cluster (TetR family transcriptional regulator). This regulator gene was associated with two genes encoding an ABC-type transport system ([GenBank: HSM_1192] and [GenBank:HSM_1193]). The closest homologs of [GenBank:HSM_1191] were found among members of Firmicutes, Spirochaetes, and Fusobacteria (e. g., [GenBank:EFM38698] encoding a putative TetR family transcriptional regulator in Eubacterium yurii subsp. margaretiae, 99\% identity, E-value = 1e-104), but not the Pasteurellaceae. The two MerR homologs of strain 2336 had only $39 \%$ identity to each other $(E-v a l u e=7 e-23)$ and both were conserved in members of the Pasteurellaceae (e.g., [GenBank:HAPS_1832] from H. parasuis and [GenBank: PM1941] from P. multocida, 85-99\%\% identity, E-values = $4 \mathrm{e}-57$ to $4 \mathrm{e}-70)$. However, the MerR homologs have been included in different protein clusters ([GenBank: HSM_1728] in [GenBank:CLSK892364] and [GenBank: HSM_1741] in [GenBank:CLSK2299246]) and appear to regulate different functions. Since [GenBank:HSM_1728] was associated with genes involved in copper metabolism ([GenBank:HSM_1729] encodes a metal binding protein, [GenBank:HSM_1730] encodes a multicopper oxidase, and [GenBank:HSM_1731] encodes a copper-translocating ATPase), it may encode a copper efflux regulator, CueR. However, [GenBank:HSM_1741] may be involved in zinc homeostasis since it was associated with zinc-responsive genes ([GenBank:HSM_1740] encodes a zinc efflux protein and [GenBank:HSM_1739] encodes a zinc-dependent hydrolase). The NCBI protein clusters database lists [GenBank:HSM_0806] as one of the members of the [GenBank: CLSK797597] cluster (LysR family transcriptional regulator, [GenBank:COG0583K]). The closest homologs of [GenBank:HSM_0806] were found in Pasteurella dagmatis ([GenBank:EEX49428], 65\% identity, E-value = 5e-113), H. influenzae ([GenBank:NTHI0936], 59\% identity, Evalue $=1 \mathrm{e}-101)$, and several species of Streptococci (e.g., [GenBank:SUB1393] and [GenBank:SPy_1634], 50-52\% identity, E-values $=2 \mathrm{e}-86$ to $1 \mathrm{e}-83$ ). In strain 2336, [GenBank:HSM_0817] encoded a putative OmpR family response regulator whereas [GenBank:HSM_1124] encoded a putative ArcA family response regulator. These response regulators have very low homology to each other (33\% identity, E-value = 7e-34). However, their amino-termini contained two conserved aspartic acid residues (Asp11 and Asp-56) that may serve as phosphate acceptors and their carboxy-termini contained several conserved residues that may be involved in DNA binding (data not shown). Strains 2336 and 129Pt also contained genes that encoded putative histidine kinases ([GenBank:HSM_0483] and [GenBank:HS_1510], [GenBank:HSM_0727] and [GenBank:HS_0402], and [GenBank:HSM_1378 and [GenBank:
HS_0900]). Whereas [GenBank:HSM_1378] and [GenBank:HS_0900] had an ORF downstream that encodes a putative cognate response regulator ([GenBank: HSM_1379] and [GenBank:HS_0901]), [GenBank: HSM_0727] and [GenBank:HS_0402] had an ORF downstream that encodes a transcriptional regulator with an $\mathrm{N}$ terminal XRE-type helix-turn-helix domain ([GenBank: HSM_0728] and [GenBank:HS_0403]). Strain 2336 contained an additional gene that encoded a putative histidine kinase ([GenBank:HSM_0824]) that had a homolog in P. multocida strain Pm70 ([GenBank:PM1380], 58\% identity, E-value $=0)$, but not in strain $129 \mathrm{Pt}$.

\section{Identification of genes encoding restriction-modification enzymes}

Type I and Type II restriction-modification (RM) systems comprise the most frequently encountered DNA modifying enzymes among eubacteria. Strain 2336 contained ORFs that putatively encode proteins of the Type I RM system ([GenBank:HSM_1615], hsdR, site-specific deoxyribonuclease; [GenBank:HSM_1617], hsdS, DNA specificity subunit; [GenBank:HSM_1619], hsdM, DNA methylation subunit). Although strain $129 \mathrm{Pt}$ lacked $h s d M$, it contained a full-length $h s d R$ ([GenBank:HS_0559]) and two truncated hsdS ([GenBank:HS_0554] and [GenBank:HS_0556]) orthologs. Strain 2336 also contained an operon putatively encoding enzymes of the Type II RM system ([GenBank: HSM_0801], M.hsoI, DNA-cytosine methyltransferase; [GenBank:HSM_0802], R.hsoI, restriction endonuclease). At the predicted protein level, M.HsoI has $68 \%$ identity to M.HinP1I ([GenBank:AAW33810], E-value = 2e-121) and R.HsoI has $72 \%$ identity to R.HinP1I ([GenBank: AAW33811], E-value $=3 \mathrm{e}-105)$ of $H$. influenzae P1. Strain 129Pt lacked M.hsoI and R.hsoI but contained an operon putatively encoding a BcgI-like RM system ([GenBank: HS_0430], site-specific DNA-methyltransferase; [GenBank:HS_0431], restriction enzyme; [GenBank:HS_0432], restriction enzyme; [GenBank:HS_0433], hypothetical protein) that is absent in strain 2336.

\section{Identification of other genes relevant to metabolism and survival}

Both $H$. somni strains contained ORFs encoding proteins putatively involved in the transport and metabolism of fucose ([GenBank:HSM_0580] and [GenBank:HS_1451] to [GenBank:HSM_0585] and [GenBank:HS_1446]), mannose ([GenBank:HSM_0956] and [GenBank:HS_0605] to [GenBank:HSM_0960] and [GenBank:HS_0609]), xylose ([GenBank:HSM_0933] and[GenBank:HS_0584] to [GenBank:HSM_0937] and [GenBank:HS_0588]), galactitol ([GenBank:HSM_1030] and [GenBank:HS_1146] to [GenBank:HSM_1036] and [GenBank:/HS_1140]), mannitol ([GenBank:HSM_0825] and [GenBank:HS_1252] to 
[GenBank:HSM_0827] and [GenBank:HS_1250]), and myo-inositol ([GenBank:HSM_0425] and [GenBank: HS_1586] to [GenBank:HSM_0435] and [GenBank: HS_1576]). Furthermore, strain 2336 contained a locus with three ORFs encoding proteins putatively involved in galactose utilization (galTKM; [GenBank:HSM_0108] to [GenBank:HSM_0110]), whereas strain 129Pt contained only galM ([GenBank:HS_0236]) and truncated galK ([GenBank:HS_0235]) orthologs. However, both strains have galE, and strain 129Pt has galactose in its LOS (38), indicating that it can metabolize galactose.

Strain 2336-specific genes encoding proteins putatively involved in carbohydrate metabolism included [GenBank: HSM_0818] (ribokinase-like domain-containing protein), [GenBank:HSM_0819] (ketose-bisphosphate aldolase), [GenBank:HSM_0820] (sugar-related regulatory protein), [GenBank:HSM_0821] (sugar binding protein), [GenBank:HSM_0822] (monosaccharide-transporting ATPase), and [GenBank:HSM_0823] (ABC type sugar transporter). Strain 2336 also contained ORFs encoding a cyclase family protein and a membrane-spanning protein ([GenBank:HSM_1907] and [GenBank:HSM_1908]). These ORFs appeared to form an operon with ORFs encoding proteins putatively involved in butyrate/pyruvate metabolism ([GenBank:HSM_1903] to [GenBank: HSM_1906]). Furthermore, strain 2336 contained an ORF that encoded an oligopeptide permease ABC transporter homolog ([GenBank:HSM_0695]). The putative Opp protein of strain 2336 was related to $\mathrm{OppB}$ proteins involved in peptide transport in E. coli and Bacillus subtilis (e.g., [GenBank:AAC74326] and [GenBank:OPPB_BACSU], respectively, $46-76 \% \%$ identity, E-values $=2 \mathrm{e}-$ 138 to $1 \mathrm{e}-72$ ).

GI I of strain 129Pt contained a gene encoding a putative phosphotransferase system protein ([GenBank: HS_0437], cellobiose-specific IIC component) whose homologs are found in M. haemolytica ([GenBank: MHA_2539], 94\% identity, E-value $=0$ ) and $H$. parasuis ([GenBank:HAPS_1535], 86\% identity, E-value $=0$ ). Strain 129Pt also contained a gene encoding a virulenceassociated protein E ([GenBank:HS_0427]). This protein was related to the VirE proteins encoded by genes found on Staphylococcus phage phi2958PVL ([GenBank: BAG74398], 31\% identity, E-value $=4 \mathrm{e}-36)$ and Enterococcus phage phiFL4A ([GenBank:ACZ64175], 33\% identity, E-value $=3 \mathrm{e}-31)$. Strain $129 \mathrm{Pt}$ contained genes ([GenBank:HS_0631] and [GenBank:0632]) encoding a putative serine/threonine protein kinase-phosphatase pair. Strain 129Pt also contained loci encoding proteins putatively involved in thiamine biosynthesis (thiMDE; [GenBank:HS_0090-0092]), tellurite resistance (terDEZ; [GenBank:HS_0633-0635]), and lysine degradation (cadBA; [GenBank:HS_1006 and [GenBank:HS_1007]).

\section{Discussion}

Genetic events such as deletions, duplications, insertions, and inversions are relatively common in bacterial chromosomes as a result of bacteriophage infection, integration and excision of plasmids, transpositions, and/or replication-mediated translocations [40,41]. In addition, different prophages embedded within a single chromosome can contain similar genes encoding integration and structural functions, and it is not uncommon for these genes to undergo homologous recombination. One of the consequences of such homologous recombination is the rearrangement of the host chromosome [42]. These events are known to be the precursors of evolution and can bring about a significant change in the number, linear order, and orientation of genes on the circular chromosomes of different strains/species of closely related bacteria [43].

The presence of PRs in the chromosomes of strains 2336 and $129 \mathrm{Pt}$ was a notable feature since the number and diversity of genes associated with these PRs far exceeded those described in $H$. influenzae strains $\mathrm{Rd}$ KW20 and 86-028NP [44,45]. The difference in the size of the chromosomes of strains 2336 and 129Pt was partly due to PRs and associated genes. Similar observations have been made in other bacteria wherein prophageassociated sequences constitute a large portion of strainspecific DNA [42]. Although one of the functions of RM systems is to afford protection against bacteriophage attack (the "cellular defense hypothesis"), it is interesting to note that both strains contain several prophage-like sequences despite the presence of genes encoding putative RM systems in their chromosomes. The lack of ORFs encoding HsdM, M.HsoI, and R.HsoI in strain $129 \mathrm{Pt}$ indicates that these systems are not absolutely essential for cell survival. Their absence may also partially explain the relative ease with which this strain can be transformed in the laboratory.

Biosynthesis of polysaccharides requires a multitude of GTs, which catalyze the transfer of sugars from an activated donor to an acceptor molecule and are usually specific for the glycosidic linkages created [46]. Intra and interspecies divergence of genes encoding GTs are not uncommon. Phase-variable LOS is an important virulence factor of pathogenic strains of $H$. somni. Phase-variation of $H$. somni LOS has been shown to be due to the presence of SSRs in genes that encode GTs and enzymes involved in assembling non-glycose LOS components such as phosphorylcholine $[5,7,37]$. The genes lob1, lob $2 A B$, and $l o b 2 D$ contain SSRs either just before the start codons or within the open reading frame [47]. In addition, [GenBank:HSM_0148], [GenBank:HSM_0164], [GenBank:HSM_0975], and [GenBank:HSM_1552] also contain SSRs that may be responsible for LOS phase variation, but require additional experimental investigation. 
Most $H$. somni strains also produce a biofilm-associated EPS consisting primarily of mannose and galactose [47]. Although characterization of some of the genes involved in the biosynthesis and/or modification of H. somni LOS/ EPS has been determined, the identification of several more has been facilitated by comparative genome analyses $[12,13,37]$. It is likely that some of the observed variations among genes encoding GTs in $H$. somni and other Pasteurellaceae members is due to recombination events and/or selective pressure. Furthermore, variation in the composition and structure of the LOSs of strains 2336 and $129 \mathrm{Pt}$ may, in part, be due to different GT genes they have acquired or lost. In view of this, the role of new GT genes putatively involved in LOS biosynthesis and phase variation that have been identified in this study needs to be investigated.

Several types of two-partner secretion (TPS) pathways have been identified and characterized in Gram-negative bacteria [48]. Filamentous hemagglutinins (Fha), consisting of a membrane-anchored protein (FhaC), which is involved in the activation/secretion of the cognate hemagglutinin/adhesin (FhaB), are prototypes of two-partner virulence systems. Homologs of $\mathrm{FhaB}$ and $\mathrm{FhaC}$ that possibly play a role in pathogenesis have been found in several members of the genera Bordetella, Haemophilus, Proteus, and Pasteurella, [49-53]. Among the 4 loci containing fha homologs in strain 2336, locus II appeared to be an acquisition mediated by a transposon and locus I appeared to be an acquisition due to homologous recombination. It appears that strain 129Pt has lost an fhaB homolog due to bacteriophage excision (Locus IV). It is possible that $f h a B$ homologs in locus I of strain 2336 are paralogs, as are the fhaB homologs in locus IV. Together, these genes represent a large collection of $f h a B$ and fhaC homologs in a single genome. The presence of multiple fhaB homologs in strain 2336 may, in part, be responsible for the serum resistance of this strain, in contrast to strain $129 \mathrm{Pt}$, which does not contain full length $f h a B$ homologs and is serumsensitive [10]. Structural and functional studies of N-terminal fragments of FHA from B. pertussis and HpmA from $P$. mirabilis have indicated that the proteins form a righthanded parallel $\beta$-helix [52-54]. Several residues that mediate the interaction of $B$. pertusis FHA with its cognate FhaC, and facilitate secretion, have also been identified [54]. Although H. somni FhaB homologs have some features in common with FHA of B. pertussis and HpmA of $P$. mirabilis, a distinct region is the direct repeat 2 Fic domain in the FhaB homolog in locus III of strain 2336 that has been shown to induce cytotoxicity in human HeLa cells, bovine turbinate cells, and bovine alveolar type 2 cells $[55,56]$. However, the secretion determinants of these proteins and the role of $\mathrm{FhaC}$ proteins in their secretion remain unknown.
Subtilases ([GenBank:COG1404]; subtilisin-like serine proteases) are a large superfamily of functionally diverse endo- and exo-peptidases that occur in prokaryotes and eukaryotes [57]. Bacterial subtilisins may have a role in pathogenesis besides facilitating protein degradation and nutrient acquisition [58]. However, subtilisin-like serine proteases from members of the Pasteurellaceae have not been characterized previously. The presence of a gene encoding a putative subtilase whose homologs were not found in other members of the Pasteurellaceae was yet another example of HGT in strain 2336. Although H. ducreyi strain $35000 \mathrm{HP}$ contains genes ([GenBank: HD1094] and [GenBank:HD1278]) encoding serine proteases that belong to the D-H-S family, they are unrelated to each other and to [GenBank:HSM_188]. In Agrobacterium tumefaciens, genes encoding AAA-ATPase and subtilisin-like serine protease have been shown to be functionally related and this pair has been proposed to constitute a toxin-antitoxin system that contributes to stability of plasmid pTiC58 [59]. A conjugative megaplasmidencoded subtilase has been shown to be a virulence factor in E. coli and it has been suggested this toxin activates a V-ATPase in Vero cells $[60,61]$. Whether the ATPase-subtilisin pair identified in this study is transcriptionally and functionally coupled, and whether the protease gene contributes to the pathogenicity of strain 2336 , has yet to be determined.

The ability to acquire and metabolize iron is an important determinant of bacterial survival and adaptability. In some bacteria, genes that facilitate iron uptake have been shown to be acquired by horizontal transfer [62]. Several members of the Pasteurellaceae possess special outer membrane protein (OMP) receptors consisting of two unrelated transferrin-binding proteins, TbpA and TbpB, which facilitate acquisition of transferrin-bound iron from their hosts [63]. H. somni strain 649 has a TbpA-TbpB receptor system that acquires iron only from bovine transferrin, and a second, probably redundant, TbpA2 receptor that can acquire iron from bovine, caprine, or ovine transferrins [64]. From genomic analyses, it is apparent that $H$. somni strains possess multiple genes for the acquisition of iron. Horizontal transfer and clustering of genes related to iron metabolism is indicative of enrichment and adaptation of pathogenic $H$. somni to different niches within its natural host. Furthermore, products of one or more of these genes may facilitate binding to transferrins/lactoferrins of different host species and such a gene repertoire could enhance the ability of this bacterium to survive in a variety of ruminants. Studies using a mouse model have suggested the role of bovine transferrin and lactoferrin in increasing the virulence of strain 2336 [65].

Many bacterial pathogens contain surface proteins that facilitate adhesion to and/or invasion of the host 
mucosal barriers $[66,67]$. Some of these proteins may also be involved in bacterial aggregation to form biofilms and their evasion of the host's innate immune system $[67,68]$. At least three major categories of bacterial adherence proteins have been identified, two of which are hair-like structures called pili and non-pilus-associated proteins called adhesins. The plasmid-encoded YadA is a prototype non-pilus-associated protein that has been well characterized [69]. Mutation or deletion of the yadA homolog can reduce virulence in pathogenic bacteria [70,71]. Large adhesins in some bacteria are associated with $\mathrm{ABC}$ transporters and may be involved in biofilm formation [72]. A transposon mutagenesis approach has implicated several genes, including those encoding filamentous haemagglutinins, in H. somni biofilm formation [73]. H. somni also contains genes putatively involved in adhesin synthesis/transport, pilus formation, and quorum sensing (e.g., luxS), but their role in facilitating biofilm formation remains to be investigated.

Veterinarians use several antibiotics to treat $H$. somni infections and feedlot cattle enterprises frequently rely on tetracyclines for prophylaxis as well as growth promotion $[1,74,75]$. Although not common, $H$. somni resistance to tetracycline has been reported [76,77]. Copper and zinc are often included in commercial cattle diets to achieve optimal growth and reproduction. Emergence of copper/ zinc resistance in bacteria of animal origin has been documented and attributed to the excessive presence of these metals in livestock feed [78]. Furthermore, the occurrence of genes related to metal and antibiotic resistance on integrative/conjugative elements and their horizontal co-transfer has been noted previously $[79,80]$. In view of these observations, it was not surprising to find a GI containing genes putatively involved in copper, zinc, and tetracycline resistance in strain 2336.

Transcriptional regulators play crucial roles in bacterial functions and they have been classified into a number of families [81]. The homologs of [GenBank:HSM_0806] (LysR, [NCBI:CLSK797597] cluster) in H. influenzae and $P$. dagmatis are associated with genes encoding proteins involved in fatty acid metabolism (e.g., acetyl-CoA acetyltransferase, 3-oxoacid CoA-transferase, and fatty acid transporters). Therefore, this cluster may represent a novel class of metabolic regulators within the LysR family. Most members of the [NCBI:PRK13756] cluster are involved in regulation of antibiotic resistance genes [81]. Homologs of [GenBank:HSM_1734] and [GenBank:HSM_1735] among members of the Pasteurellaceae encode tetracycline resistance and are associated with mobile genetic elements [82-86]. Homologs of [GenBank:HSM_1736] and [GenBank:HSM_1737] in other bacteria are known to be horizontally transferred and may mediate resistance to antibiotics [87]. Furthermore, homologs of [GenBank:
HSM_1192] and [GenBank:HSM_1193] are predicted to be involved in multidrug resistance $[88,89]$. In summary, it appears that strain 2336 contained at least three different systems related to antibiotic resistance. Although the functional role of these genes remains to be established, their similarity to metal/antibiotic resistance genes associated with mobile genetic elements in other members of the Pasteurellaceae is clinically significant.

From genome comparisons, it appears that there is no correlation between chromosome size and the number of tRNA genes (the genomes of $H$. somni 2336, H. somni 129Pt, H. influenzae 86-028NP, H. ducreyi 35000HP, and P. multocida Pm70 contain 49, 49, 58, 46, and 57 tRNA genes, respectively). Whether the lower number of tRNA genes found in $H$. somni strains is due to disruptive integration of bacteriophages into tRNA genes (as in "bacteriophage disruption of tRNA genes in Lactobacillus johnsonii' [90]) or is a result of compensatory gene loss in lieu of acquisition of new genes (as in 'genome reduction in pathogenic and symbiotic bacteria' [91]) is unknown. Nevertheless, comparison of the chromosomes of strains 129Pt and 2336 bolsters the proposition that prophages and transposons have played a major role in creating genomic diversity and phenotypic variability in the two strains. It is also apparent that strains 2336 and $129 \mathrm{Pt}$ have independently and intermittently acquired and lost genes since their divergence from a common ancestor, and that the net gain in strain 129Pt is less than the net gain in strain 2336.

\section{Conclusions}

H. somni strain 2336 contains a larger chromosome when compared to other Haemophilus and Histophilus strains whose genome sequences are available. Several regions that resemble the pathogenicity islands of other virulent bacteria are present in strain 2336. There is evidence to suggest that most of these regions were acquired by HGT mechanisms, whereas similar regions were not found in the commensal strain 129Pt. Although previous studies have discovered the genetic basis for some of the phenotypic dissimilarities between strains 2336 and $129 \mathrm{Pt}$, complete genome sequence analyses have provided a comprehensive account of innate and acquired genetic traits. Furthermore, comparisons of the genomes of strains 2336 and 129Pt have contributed to our understanding of the biology and pathogenic evolution of these bacteria. The post-genomic era for $H$. somni poses new challenges and opportunities in terms of functional characterization of genes and deciphering their roles in colonization, survi$\mathrm{val}$, and pathogenesis. Continued analyses of the genomes of $H$. somni strains and comparison with newly sequenced genomes of other bacteria should enhance the current knowledge on virulence mechanisms. Nevertheless, the results from this study are expected to facilitate the 
development of improved diagnostic tests for and vaccines against $H$. somni.

\section{Additional material}

Additional file 1: Additional file 1 List of $H$. somni strain 2336 specific genes. This table lists the strain-specific genes found in $\mathrm{H}$. somni strain 2336. This data was obtained by cross-comparison of the genomes of strains 2336 and 129Pt using blastn.

Additional file 2: Additional file 2 List of $\mathrm{H}$. somni strain 129Pt specific genes. This table lists the strain-specific genes found in $\mathrm{H}$. somni strain 129Pt. This data was obtained by cross-comparison of the genomes of strains 129Pt and 2336 using blastn.

\begin{abstract}
Acknowledgements
This work was performed under the auspices of the US Department of Energy's Office of Science, Biological and Environmental Research Program and by the University of California, Lawrence Livermore National Laboratory under Contract No. DE-AC02-05CH1 1231, Lawrence Livermore National Laboratory under Contract No. DE-AC52-07NA27344, and Los Alamos National Laboratory under contract No. DE-AC02-06NA25396. This work was supported by U.S. Department of Agriculture (Cooperative State Research, Education, and Extension Service, Initiative for Future Agriculture and Food Systems) grants 2001-52100-11314, 2003-35204-13637, 2007-35204-18338 to TII, the U.S. Department of Energy under contract no. W-7405-ENG-36, and funds from the Virginia Agricultural Experiment Station. We thank Gretchen Berg, Dr. Michael Howard, and Dr. Shaadi Elswaifi for technical assistance.
\end{abstract}

\section{Author details}

${ }^{1}$ Center for Molecular Medicine and Infectious Diseases, Virginia-Maryland Regional College of Veterinary Medicine, Virginia Polytechnic Institute and State University, Blacksburg, Virginia 24061, USA. ²DOE Joint Genome Institute, Los Alamos National Laboratory, Los Alamos, New Mexico 87545, USA. ${ }^{3}$ Laboratory for Genomics and Bioinformatics, and Department of Microbiology and Immunology, University of Oklahoma Health Sciences Center, Oklahoma City, Oklahoma 731042, USA.

\section{Authors' contributions}

SS performed the annotation using RAST, planned the comparative analysis, generated all the figures, and drafted most of the manuscript. JFC, AJD, and AFG contributed to whole genome comparisons and identification of putative virulence genes. $M C, J G, J O, J Z$, and $G B$ contributed to the whole genome shotgun sequencing of strain 2336 at OUHSC. DB, OC, JCD, CSH, and RT contributed to the finishing of the genome of strain 2336 at LANL and participated in the study design and comparative analysis of the two genomes. TII and DWD conceived the study, participated in genome analyses, and helped draft the manuscript. All authors read and approved the final manuscript.

Received: 15 June 2011 Accepted: 23 November 2011 Published: 23 November 2011

\section{References}

1. Inzana TJ: The Haemophilus somnus complex. In Current Veterinary Therapy 4.. 4 edition. Edited by: Howard JL, Smith RA. Philadelphia, PA: W.B. Saunders Co.; 1999:358-361.

2. Corbeil LB: Histophilus somni host-parasite relationships. Anim Health Res Rev 2007, 8:151-160.

3. Widders PR, Dorrance LA, Yarnall M, Corbeil LB: Immunoglobulin-binding activity among pathogenic and carrier isolates of Haemophilus somnus. Infect Immun 1989, 57:639-642.

4. Corbeil L, Gogolewski R, Stephens L, Inzana T: Haemophilus somnus: antigen analysis and immune responses. In Haemophilus, Actinobacillus, and Pasteurellal. Edited by: Donachie W, Lainson F, Hodgson J. New York: Plenum Press; 1995:63-73.
5. Wu Y, McQuiston JH, Cox A, Pack TD, Inzana TJ: Molecular cloning and mutagenesis of a DNA locus involved in lipooligosaccharide biosynthesis in Haemophilus somnus. Infect Immun 2000, 68:310-319.

6. Cole SP, Guiney DG, Corbeil LB: Molecular analysis of a gene encoding a serum-resistance-associated $76 \mathrm{kDa}$ surface antigen of Haemophilus somnus. J Gen Microbiol 1993, 139:2135-2143.

7. McQuiston JH, McQuiston JR, Cox AD, Wu Y, Boyle SM, Inzana TJ: Characterization of a DNA region containing 5'-(CAAT)(n)-3' DNA sequences involved in lipooligosaccharide biosynthesis in Haemophilus somnus. Microb Pathog 2000, 28:301-312.

8. Inzana TJ, Gogolewski RP, Corbeil LB: Phenotypic phase variation in Haemophilus somnus lipooligosaccharide during bovine pneumonia and after in vitro passage. Infect Immun 1992, 60:2943-2951.

9. Inzana TJ, Glindemann G, Cox AD, Wakarchuk W, Howard MD: Incorporation of $\mathrm{N}$-acetylneuraminic acid into Haemophilus somnus lipooligosaccharide (LOS): enhancement of resistance to serum and reduction of LOS antibody binding. Infect Immun 2002, 70:4870-4879.

10. Corbeil LB, Bastida-Corcuera FD, Beveridge TJ: Haemophilus somnus immunoglobulin binding proteins and surface fibrils. Infect Immun 1997, 65:4250-4257.

11. Fraser CM, Eisen J, Fleischmann RD, Ketchum KA, Peterson S: Comparative genomics and understanding of microbial biology. Emerg Infect Dis 2000, 6:505-512

12. Challacombe JF, Inzana TJ: Comparative genomics of Pasteurellaceae. In Pasteurellaceae: Biology, Genomics, and Molecular Aspects $\backslash$. Edited by: Kuhnert P, Christensen H. Norfolk, UK: Caister Academic Press; 2008:53-77.

13. Challacombe JF, Duncan AJ, Brettin TS, Bruce D, Chertkov O, Detter JC, Han CS, Misra M, Richardson P, Tapia R, Thayer N, Xie G, Inzana TJ: Complete genome sequence of Haemophilus somnus (Histophilus somni) strain 129Pt and comparison to Haemophilus ducreyi $35000 \mathrm{HP}$ and Haemophilus influenzae Rd. J Bacteriol 2007, 189:1890-1898.

14. Hacker J, Blum-Oehler G, Muhldorfer I, Tschape H: Pathogenicity islands of virulent bacteria: structure, function and impact on microbial evolution. Mol Microbiol 1997, 23:1089-1097.

15. Olson M: When less is more: gene loss as an engine of evolutionary change. Am J Hum Genet 1999, 64:18-23.

16. St Michael F, Li J, Howard MD, Duncan AJ, Inzana TJ, Cox AD: Structural analysis of the oligosaccharide of Histophilus somni (Haemophilus somnus) strain 2336 and identification of several lipooligosaccharide biosynthesis gene homologues. Carbohydr Res 2005, 340:665-672.

17. Corbeil LB, Blau K, Prieur DJ, Ward AC: Serum susceptibility of Haemophilus somnus from bovine clinical cases and carriers. J Clin Microbiol 1985, 22(2):192-198.

18. Lander ES, Waterman MS: Genomic mapping by fingerprinting random clones: a mathematical analysis. Genomics 1988, 2:231-239.

19. Chissoe SL, Wang YF, Clifton SW, Ma N, Sun HJ, Lobsinger JS, Kenton SM, White JD, Roe BA: Strategies for rapid and accurate DNA sequencing. Methods: Companion Methods Enzymol 1991, 3:55-65.

20. Munson RS, Harrison A, Gillaspy A, Ray WC, Carson M, Armbruster D, Gipson J, Gipson M, Johnson L, Lewis L, Dyer DW, Bakaletz LO: Partial analysis of the genomes of two nontypeable Haemophilus influenzae otitis media isolates. Infect Immun 2004, 72:3002-3010.

21. Karlyshev AV, Pallen MJ, Wren BW: Single-primer PCR procedure for rapid identification of transposon insertion sites. Biotechniques 2000, 28:1078.

22. Gordon D, Desmarais C, Green P: Automated finishing with autofinish. Genome Res 2001, 11:614-625.

23. Han CS, Chain P: Finishing repeat regions automatically with Dupfinisher. In Proc 2006 Int Conf Bioinformatics Comput Bio/. Edited by: Arabnia HR, and H. Valafar. Las Vegas, NV.: CSREA Press; 2006:141-146.

24. Anonymous: Transposon-based strategies for efficient DNA sequencing and functional genomics (available online at [http://www.epibio.com/ forum_issue.asp]. EPICENTRE Forum 2004, 11:22-23.

25. Finn RD, Mistry J, Schuster-Bockler B, Griffiths-Jones S, Hollich V, Lassmann T, Moxon S, Marshall M, Khanna A, Durbin R, Eddy SR, Sonnhammer ELL, Bateman A: Pfam: clans, web tools and services. Nucleic Acids Res 2006, 34 Database: D247-D251.

26. Smith TF, Waterman MS: Identification of common molecular subsequences. J Mol Biol 1981, 147:195-197.

27. Aziz RK, Bartels D, Best AA, DeJongh M, Disz T, Edwards RA, Formsma K, Gerdes S, Glass EM, Kubal M, Meyer F, Olsen GJ, Olson R, Osterman AL, 
Overbeek RA, McNeil LK, Paarmann D, Paczian T, Parrello B, Pusch GD, Reich C, Stevens R, Vassieva O, Vonstein V, Wilke A, Zagnitko O: The RAST Server: rapid annotations using subsystems technology. BMC Genomics 2008, 9:75.

28. Darling AC, Mau B, Blattner FR, Perna NT: Mauve: multiple alignment of conserved genomic sequence with rearrangements. Genome Res 2004, 14:1394-1403.

29. Darling AE, Treangen TJ, Messeguer X, Perna NT: Analyzing patterns of microbial evolution using the mauve genome alignment system. Methods Mol Biol 2007, 396:135-152.

30. Delcher AL, Phillippy A, Carlton J, Salzberg SL: Fast algorithms for largescale genome alignment and comparison. Nucleic Acids Res 2002, 30:2478-2483

31. Lima-Mendez G, Van Helden J, Toussaint A, Leplae R: Prophinder: a computational tool for prophage prediction in prokaryotic genomes. Bioinform 2008, 24:863-865.

32. Langille MG, Hsiao WW, Brinkman FS: Detecting genomic islands using bioinformatics approaches. Nature Rev Microbiol 2010, 8:373-382.

33. Markowitz VM, Chen IM, Palaniappan K, Chu K, Szeto E, Grechkin Y, Ratner A, Anderson I, Lykidis A, Mavromatis K, Ivanova NN, Kyrpides NC: The integrated microbial genomes system: an expanding comparative analysis resource. Nucleic Acids Res 2010, , 38 Database: D382-D390.

34. Siddaramappa S, Duncan AJ, Brettin T, Inzana TJ: Comparative analyses of two cryptic plasmids from Haemophilus somnus (Histophilus somni). Plasmid 2006, 55:227-234.

35. Feschotte C: Merlin, a new superfamily of DNA transposons identified in diverse animal genomes and related to bacterial IS1016 insertion sequences. Mol Biol Evol 2004, 21:1769-1780.

36. Siguier P, Gagnevin L, Chandler M: The new IS1595 family, its relation to IS1 and the frontier between insertion sequences and transposons. Res Microbiol 2009, 160:232-241.

37. Elswaifi SF, St Michael F, Sreenivas A, Cox A, Carman GM, Inzana TJ: Molecular characterization of phosphorylcholine expression on the lipooligosaccharide of Histophilus somni. Microb Pathog 2009, 47:223-230.

38. St Michael F, Howard MD, Li J, Duncan AJ, Inzana TJ, Cox AD: Structural analysis of the lipooligosaccharide from the commensal Haemophilus somnus genome strain 129Pt. Carbohydr Res 2004, 339:529-535.

39. Ward CK, Lumbley SR, Latimer JL, Cope LD, Hansen EJ: Haemophilus ducreyi secretes a filamentous hemagglutinin-like protein. J Bacteriol 1998, 180:6013-6022.

40. Tillier ER, Collins RA: Genome rearrangement by replication-directed translocation. Nat Genet 2000, 26:195-197

41. Brussow H, Canchaya C, Hardt WD: Phages and the evolution of bacterial pathogens: from genomic rearrangements to lysogenic conversion. Microbiol Mol Biol Rev 2004, 68:560-602.

42. Canchaya C, Fournous $\mathrm{G}$, Brussow $\mathrm{H}$ : The impact of prophages on bacterial chromosomes. Mol Microbiol 2004, 53:9-18.

43. Moret BME, Bader DA, Warnow T: High-performance algorithm engineering for computational phylogenetics. J Supercomput 2002, 22:99-111.

44. Harrison A, Dyer DW, Gillaspy A, Ray WC, Mungur R, Carson MB, Zhong H, Gipson J, Gipson M, Johnson LS, Lewis L, Bakaletz LO, Munson RS Jr: Genomic sequence of an otitis media isolate of nontypeable Haemophilus influenzae: comparative study with $\mathrm{H}$. influenzae serotype d, strain KW20. J Bacteriol 2005, 187:4627-4636.

45. Fleischmann RD, Adams MD, White O, Clayton RA, Kirkness EF, Kerlavage AR, Bult CJ, Tomb JF, Dougherty BA, Merrick JM, Sutton G, FitzHugh W, Fields C, Gocayne JD, Scott J, Shirley R, Liu L-I, Glodek A, Kelly JM, Weidman JF, Phillips CA, Spriggs T, Hadblom E, Cotton MD, Utterback TR, Hanna MC, Nguyen DT, Saudek DM, Brandon RC, Fine LD, et al: Whole-genome random sequencing and assembly of Haemophilus influenzae Rd. Science 1995, 269:496-512.

46. Lundborg M, Modhukur V, Widmalm G: Glycosyltransferase functions of $E$. coli O-antigens. Glycobiol 20:366-368.

47. Inzana TJ, Swords WE, Sandal I, Siddaramappa S: Lipopolysaccharides, biofilms and quorum sensing in Pasteurellaceae. In Pasteurellaceae: Biology, Genomics and Molecular Aspects $\backslash$. Edited by: Kuhnert P, Christensen H. Norfolk, UK: Caister Academic Press; 2008:177-195.

48. Jacob-Dubuisson F, Locht C, Antoine R: Two-partner secretion in Gramnegative bacteria: a thrifty, specific pathway for large virulence proteins. Mol Microbiol 2001, 40:306-313.
49. Fuller TE, Kennedy MJ, Lowery DE: Identification of Pasteurella multocida virulence genes in a septicemic mouse model using signature-tagged mutagenesis. Microb Pathog 2000, 29:25-38.

50. Ward CK, Mock JR, Hansen EJ: The LspB protein is involved in the secretion of the LspA1 and LspA2 proteins by Haemophilus ducreyi. Infect Immun 2004, 72:1874-1884.

51. May BJ, Zhang Q, Li LL, Paustian ML, Whittam TS, Kapur V: Complete genomic sequence of Pasteurella multocida, Pm70. Proc Natl Acad Sci USA 2001, 98:3460-3465.

52. Clantin B, Hodak H, Willery E, Locht C, Jacob-Dubuisson F, Villeret V: The crystal structure of filamentous hemagglutinin secretion domain and its implications for the two-partner secretion pathway. Proc Natl Acad Sci USA 2004, 101:6194-6199.

53. Weaver TM, Hocking JM, Bailey LJ, Wawrzyn GT, Howard DR, Sikkink LA, Ramirez-Alvarado M, Thompson JR: Structural and functional studies of truncated hemolysin A from Proteus mirabilis. J Biol Chem 2009, 284:22297-22309.

54. Hodak H, Clantin B, Willery E, Villeret V, Locht C, Jacob-Dubuisson F: Secretion signal of the filamentous haemagglutinin, a model twopartner secretion substrate. Mol Microbiol 2006, 61:368-382.

55. Worby CA, Mattoo S, Kruger RP, Corbeil LB, Koller A, Mendez JC, Zekarias B, Lazar C, Dixon JE: The fic domain: regulation of cell signaling by adenylylation. Mol Cell 2009, 34:93-103.

56. Zekarias B, Mattoo S, Worby C, Lehmann J, Rosenbusch RF, Corbeil LB: Histophilus somni IbpA DR2/Fic in virulence and immunoprotection at the natural host alveolar epithelial barrier. Infect Immun 2010, 78:1850-1858.

57. Siezen RJ, Leunissen JA: Subtilases: the superfamily of subtilisin-like serine proteases. Protein Sci 1997, 6:501-523.

58. Siezen RJ, Renckens B, Boekhorst J: Evolution of prokaryotic subtilases: genome-wide analysis reveals novel subfamilies with different catalytic residues. Proteins 2007, 67:681-694.

59. Yamamoto S, Kiyokawa K, Tanaka K, Moriguchi K, Suzuki K: Novel toxinantitoxin system composed of serine protease and AAA-ATPase homologues determines the high level of stability and incompatibility of the tumor-inducing plasmid pTiC58. J Bacteriol 2009, 191:4656-4666.

60. Paton AW, Srimanote P, Talbot UM, Wang H, Paton JC: A new family of potent $A B(5)$ cytotoxins produced by Shiga toxigenic Escherichia coli. J Exp Med 2004, 200:35-46

61. Yahiro K, Morinaga N, Satoh M, Matsuura G, Tomonaga T, Nomura F, Moss J, Noda M: Identification and characterization of receptors for vacuolating activity of subtilase cytotoxin. Mol Microbiol 2006, 62:480-490.

62. Dobrindt U, Hochhut B, Hentschel U, Hacker J: Genomic islands in pathogenic and environmental microorganisms. Nat Rev Microbiol 2004, 2:414-424

63. Cornelissen CN: Transferrin-iron uptake by Gram-negative bacteria. Front Biosci 2003, 8:d836-847.

64. Ekins A, Bahrami F, Sijercic A, Maret D, Niven DF: Haemophilus somnus possesses two systems for acquisition of transferrin-bound iron. $J$ Bacteriol 2004, 186:4407-4411.

65. Geertsema RS, Kimball RA, Corbeil LB: Bovine plasma proteins increase virulence of Haemophilus somnus in mice. Microb Pathog 2007, 42:22-28.

66. Hultgren SJ, Abraham S, Caparon M, Falk P, St Geme JW, Normark S: Pilus and nonpilus bacterial adhesins: assembly and function in cell recognition. Cell 1993, 73:887-901.

67. Kline KA, Falker S, Dahlberg S, Normark S, Henriques-Normark B: Bacterial adhesins in host-microbe interactions. Cell Host Microbe 2009, 5:580-592.

68. Jurcisek JA, Bakaletz LO: Biofilms formed by nontypeable Haemophilus influenzae in vivo contain both double-stranded DNA and type IV pilin protein. J Bacterio/ 2007, 189:3868-3875.

69. Hoiczyk E, Roggenkamp A, Reichenbecher M, Lupas A, Heesemann J: Structure and sequence analysis of Yersinia YadA and Moraxella UspAs reveal a novel class of adhesins. EMBO J 2000, 19:5989-5999.

70. Heise $T$, Dersch $P$ : Identification of a domain in Yersinia virulence factor YadA that is crucial for extracellular matrix-specific cell adhesion and uptake. Proc Natl Acad Sci USA 2006, 103:3375-3380.

71. Lawrenz MB, Lenz JD, Miller VL: A novel autotransporter adhesin is required for efficient colonization during bubonic plague. Infect Immun 2009, 77:317-326.

72. Gerlach RG, Hensel M: Protein secretion systems and adhesins: the molecular armory of Gram-negative pathogens. Int J Med Microbiol 2007, 297:401-415. 
73. Sandal I, Shao JQ, Annadata S, Apicella MA, Boye M, Jensen TK, Saunders GK, Inzana TJ: Histophilus somni biofilm formation in cardiopulmonary tissue of the bovine host following respiratory challenge. Microbes Infect 2009, 11:254-263.

74. Smith MS, Yang RK, Knapp CW, Niu Y, Peak N, Hanfelt MM, Galland JC, Graham DW: Quantification of tetracycline resistance genes in feedlot lagoons by real-time PCR. Appl Environ Microbiol 2004, 70:7372-7377.

75. Van Donkersgoed J, Janzen ED, Potter AA, Harland RJ: The occurrence of Haemophilus somnus in feedlot calves and its control by postarrival prophylactic mass medication. Can Vet J 1994, 35:573-580.

76. Watts JL, Yancey RJ, Salmon SA, Case CA: A 4-year survey of antimicrobial susceptibility trends for isolates from cattle with bovine respiratory disease in North America. J Clin Microbiol 1994, 32:725-731.

77. Mohd-Zain Z, Turner SL, Cerdeno-Tarraga AM, Lilley AK, Inzana TJ, Duncan AJ, Harding RM, Hood DW, Peto TE, Crook DW: Transferable antibiotic resistance elements in Haemophilus influenzae share a common evolutionary origin with a diverse family of syntenic genomic islands. J Bacteriol 2004, 186:8114-8122.

78. Hasman $\mathrm{H}$, Franke $\mathrm{S}$, Rensing $\mathrm{C}$ : Resistance to metals used in agriculture production. In Antimicrobial resistance in bacteria of animal origin \. Edited by: Aarestrup F. Washington, DC.: ASM Press; 2006:

79. Gilmour MW, Thomson NR, Sanders M, Parkhill J, Taylor DE: The complete nucleotide sequence of the resistance plasmid R478: defining the backbone components of incompatibility group $\mathrm{H}$ conjugative plasmids through comparative genomics. Plasmid 2004, 52:182-202.

80. Baker-Austin C, Wright MS, Stepanauskas R, McArthur JV: Co-selection of antibiotic and metal resistance. Trends Microbiol 2006, 14:176-182.

81. Ramos JL, Martinez-Bueno M, Molina-Henares AJ, Teran W, Watanabe K, Zhang X, Gallegos MT, Brennan R, Tobes R: The TetR family of transcriptional repressors. Microbiol Mol Biol Rev 2005, 69:326-356.

82. Blanco M, Kadlec K, Gutierrez Martin CB, de la Fuente AJ, Schwarz S, Navas J: Nucleotide sequence and transfer properties of two novel types of Actinobacillus pleuropneumoniae plasmids carrying the tetracycline resistance gene tet(H). J Antimicrob Chemother 2007, 60:864-867.

83. Kehrenberg C, Werckenthin C, Schwarz S: Tn5706, a transposon-like element from Pasteurella multocida mediating tetracycline resistance. Antimicrob Agents Chemother 1998, 42:2116-2118.

84. Hansen LM, McMurry LM, Levy SB, Hirsh DC: A new tetracycline resistance determinant, Tet $\mathrm{H}$, from Pasteurella multocida specifying active efflux of tetracycline. Antimicrob Agents Chemother 1993, 37:2699-2705.

85. Kehrenberg C, Schwarz S: Identification of a truncated, but functionally active tet $(\mathrm{H})$ tetracycline resistance gene in Pasteurella aerogenes and Pasteurella multocida. FEMS Microbiol Lett 2000, 188:191-195.

86. Kehrenberg C, Salmon SA, Watts JL, Schwarz S: Tetracycline resistance genes in isolates of Pasteurella multocida, Mannheimia haemolytica, Mannheimia glucosida and Mannheimia varigena from bovine and swine respiratory disease: intergeneric spread of the tet $(\mathrm{H})$ plasmid pMHT1. J Antimicrob Chemother 2001, 48:631-640.

87. Bay DC, Turner RJ: Diversity and evolution of the small multidrug resistance protein family. BMC Evol Biol 2009, 9:140.

88. Saier MH, Paulsen IT, Sliwinski MK, Pao SS, Skurray RA, Nikaido H: Evolutionary origins of multidrug and drug-specific efflux pumps in bacteria. FASEB J 1998, 12:265-274.

89. Young J, Holland IB: $A B C$ transporters: bacterial exporters-revisited five years on. Biochim Biophys Acta 1999, 1461:177-200.

90. Ventura M, Canchaya C, Pridmore D, Berger B, Brussow H: Integration and distribution of Lactobacillus johnsonii prophages. J Bacteriol 2003, 185:4603-4608.

91. Hacker J, Hentschel U, Dobrindt U: Prokaryotic chromosomes and disease. Science 2003, 301:790-793.

doi:10.1186/1471-2164-12-570

Cite this article as: Siddaramappa et al.: Horizontal gene transfer in Histophilus somni and its role in the evolution of pathogenic strain 2336, as determined by comparative genomic analyses. BMC Genomics 2011 $12: 570$

\section{Submit your next manuscript to BioMed Central and take full advantage of:}

- Convenient online submission

- Thorough peer review

- No space constraints or color figure charges

- Immediate publication on acceptance

- Inclusion in PubMed, CAS, Scopus and Google Scholar

- Research which is freely available for redistribution 